September 19, 2002

\title{
PATTERNS AND MINIMAL DYNAMICS FOR GRAPH MAPS
}

\author{
LLUÍS ALSEDÀ, FRANÇOIS GAUTERO, JOHN GUASCHI, JÉRÔME LOS, \\ FRANCESC MAÑOSAS, AND PERE MUMBRÚ
}

\begin{abstract}
We study the rigidity problem for periodic orbits of (continuous) graph maps belonging to the same homotopy equivalence class. Since the underlying spaces are not necessarily homeomorphic, we define a new notion of pattern which enables us to compare periodic orbits of self-maps of homotopyequivalent spaces. This definition unifies the known notions of pattern for other spaces. The two main results of the paper are: given a free group endomorphism, we study the persistence under homotopy of the periodic orbits of its topological representatives, and in the irreducible case, we prove the minimality (within the homotopy class) of the set of periodic orbits of its efficient representatives.
\end{abstract}

\section{INTRODUCTION}

In this paper, we shall study the phenomenon of rigidity of the dynamics of graph maps. The notion of rigidity is often associated with the existence of a canonical representative within a well-defined class of objects. This is the case, for example, in hyperbolic geometry (Mostow [20]), and for surface homeomorphisms (NielsenThurston $[23,11]$ ). In each of these cases, there exists a unique (up to conjugacy) canonical representative which satisfies many extremal dynamical properties, such as minimisation of the growth rate (Besson-Courtois-Gallot [6] for hyperbolic manifolds and Fathi-Shub [11] for pseudo-Anosov homeomorphisms), and minimisation of the number of closed geodesics for hyperbolic manifolds and of periodic orbits for pseudo-Anosov homeomorphisms (Asimov-Franks [4] and T. Hall [12]) in their respective classes.

With the aim of comparing periodic orbits of different maps, the notion of pattern was introduced for each of the following important classes of maps:

- continuous maps of the interval, of the circle, and of 'fixed' graphs (where the notion of pattern is termed action) $[5,2,3]$,

- continuous maps of (finite) trees [1],

- surface homeomorphisms $[8,18,16]$ (where the notion of pattern is usually termed braid type).

The basic phenomenon that these notions of pattern are designed to encapsulate is that of coexistence or forcing of periodic orbits. The original motivation for this stemmed from Sharkovski1's theorem in 1964 for interval maps [22], which roughly speaking, states that the existence of a single periodic orbit $P$ of a given period $n$ is enough to imply the existence of other periodic orbits and often of infinitely many orbits. This result may be refined by considering the permutation

2000 Mathematics Subject Classification. Primary 37E30, 37E25, 37E15.

Key words and phrases. Graph maps, minimal dynamics, efficient representatives, combinatorial patterns.

This collaboration has been partially supported by the grant HF 1999-0046, the DGES grant PB96-1153, the CTP project Complex Analysis and Dynamics, the PICS project Complex Analysis: Function Theory and Dynamics, the CONACIT grant 1999SGR-00349, the CIRIT grant 2000SGR-00027 and the Marie Curie grant HPMF-CT-1999-00160. 
$\sigma \in S_{n}$ induced by the map on the points of $P$, the points being ordered by the natural ordering of the interval. Each permutation $\sigma$ may be interpreted as a subset of $\mathcal{C}(I, I)$, namely those continuous maps of the interval $I$ which admit a periodic orbit whose associated permutation is $\sigma$. This subset is essentially (up to homeomorphism) a relative homotopy equivalence class in $\mathcal{C}(I, I)$, relative to the periodic orbit in question. It possesses a unique (up to homeomorphism) canonical representative (the piecewise linear or 'connect-the-dots' map) which minimises the topological entropy as well as the set of periodic orbits [2]. As we pointed out above, a canonical representative with analogous minimisation properties also exists in the case of surface homeomorphisms, namely, pseudo-Anosov homeomorphisms.

The goal of this paper is to elucidate the rigidity problem for periodic orbits of (continuous) graph maps. In order to compare such orbits, it will be convenient to suppose that the fundamental groups of the graphs in question have the same rank, and that the endomorphisms induced by the maps on the fundamental groups are conjugate. It is thus natural to consider graph maps that belong to the same homotopy equivalence class. In doing so, we come up against a preliminary problem, being that the underlying spaces are not necessarily homeomorphic (this was already the case in [1]). We solve this by defining a new notion of pattern which enables us to compare periodic orbits of self-maps of homotopy-equivalent spaces, not just of graphs. Moreover, our definition in some sense unifies the above-mentioned notions of pattern for self-maps of the interval, the circle and 'fixed' graphs, for surface homeomorphisms, and finally for continuous self-maps of trees (see Remark 2.10).

A priori, given the definition of a pattern as a relative homotopy equivalence class, it is not an easy matter to check that two orbits have the same pattern. However, the combinatorial characterisation of the notion of pattern as a permutation in the case of interval maps, or for surface homeomorphisms, as a conjugacy class in the mapping class group, facilitates greatly the comparison of patterns. In the case of graph maps, we show that our notion of pattern may also be characterised combinatorially, in terms of the induced action on the fundamental groupoid of the graph "marked" by the periodic orbit, which is again a conjugacy problem.

Let us remark that in all of the above-mentioned classes of maps, the study of the minimality of the periodic orbit structure, as well as the topological entropy, may be reduced to that of a particular class of graph maps. For the interval and the circle, it is not necessary to change the class of maps under consideration; for surface homeomorphisms, the maps in question are the so-called train track maps, originally due to Williams [24] and then reintroduced by Thurston. A particular homeomorphism may be represented by different train track maps supported on non-homeomorphic graphs, but with the same homotopy type. In this framework, our definition of pattern (where the graph is not fixed) is natural.

In order to obtain a rigidity result for periodic orbits, in the final section we shall restrict our attention to the subclass of graph maps that induce irreducible free group endomorphisms of the fundamental group. The reason for this is that there exist natural candidates for the canonical representatives in the corresponding class of graph maps, namely the train track or efficient representatives. For irreducible free group automorphisms, the existence of efficient representatives follows from results of Bestvina-Handel [7] and Los [15], and for irreducible free group endomorphisms, from those of Dicks-Ventura [10]. An efficient representative is known to minimise the growth rate (or topological entropy) in its homotopy equivalence class, but little is known about the persistence and minimality properties of the set of periodic orbits of an efficient representative. 
Our goal in this paper is twofold. Given a free group endomorphism, we first study the persistence of patterns among its representatives. Secondly, in the irreducible case, we prove the minimality (within the homotopy equivalence class) of the set of periodic orbits of its efficient representatives. This will follow from the persistence result.

In order to state our main results, let us make some definitions. Given an endomorphism $\Phi$ of a free group of finite rank, a representative for $\Phi$ is defined to be a graph map such that the induced map on the fundamental group is $\Phi$, up to an inner automorphism and up to conjugacy. In the literature, one encounters the more restrictive notion of topological representative which is a representative sending vertices to vertices and edges to edge paths.

Nielsen fixed point theory and the notion of index will play an important rôle in our work. If $C$ is a Nielsen fixed point class of $f$ then $\operatorname{ind}(C, f)$ will denote its index (see [13, Section I.3]), and if ind $(C, f) \neq 0$ then $C$ will be called an essential class of $f$. A periodic orbit $P$ will be called essential if $\operatorname{ind}\left(C, f^{|P|}\right) \neq 0$, where $C$ is a fixed point class of $f^{|P|}$ containing a point of $P$, and $|P|$ denotes the period of $P$.

We now define our notion of pattern. Let $f: G \longrightarrow G$ and $g: G^{\prime} \longrightarrow G^{\prime}$ be graph maps, and let $P$ and $Q$ be periodic orbits of $f$ and $g$ respectively. We say that the triple $(G, P, f)$ is equivalent to $\left(G^{\prime}, Q, g\right)$ if there exists a homotopy equivalence $r: G \longrightarrow G^{\prime}$ such that $r \circ f \simeq_{P} g \circ r$, where $\simeq_{P}$ is a homotopy relative to $P$, and $\left.r\right|_{P}$ is a bijection of $P$ onto $Q$. The corresponding equivalence class of $(G, P, f)$, denoted by $[G, P, f]$, will be called its pattern. If in the above definition we replace the assumption that $\left.r\right|_{P}$ is a bijection by the conditions $r(P)=Q$ and $|P|>|Q|$, then we say that $\left[G^{\prime}, Q, g\right]$ is a reduction of $[G, P, f]$.

As for patterns, the notion of reduction may be characterised combinatorially in terms of the induced action on the fundamental groupoid of the graphs marked by the periodic orbits (see Section 3). Furthermore, it may also be characterised in terms of Nielsen equivalence. Indeed, $[G, P, f]$ is reducible if and only if $P$ may be partitioned into $m$ subsets of equal cardinality, each subset being contained in a fixed point class of $f^{|P|}$, and there exists a Nielsen path joining two points of the same group whose concatenation with its images under $f^{m}$ forms a homotopicallytrivial loop (see Proposition 3.3).

The following theorem summarises the basic persistence properties for patterns.

Theorem A. Let $f: G \longrightarrow G$ and $g: G^{\prime} \longrightarrow G^{\prime}$ be representatives of an endomorphism of a free group of finite rank. Then:

(a) there exists an index-preserving bijection $\kappa$ that, for each $n \in \mathbb{N}$, sends essential fixed point classes of $f^{n}$ to essential fixed point classes of $g^{n}$.

(b) let $P$ be an essential periodic orbit of $f$, let $C$ be the fixed point class for $f^{|P|}$ of a point of $P$, and let $Q$ be the $g$-orbit of a point of $\kappa(C)$. Then either $\left[G^{\prime}, Q, g\right]=[G, P, f]$, or $\left[G^{\prime}, Q, g\right]$ is a reduction of $[G, P, f]$.

As we already pointed out, from this theorem we obtain a minimality result for the set of periodic orbits of efficient representatives of irreducible free group endomorphisms. In order to state this result precisely, we introduce some more definitions.

Let $\mathbb{F}_{n}$ denote the free group of rank $n$, and let $\Phi$ be an endomorphism of $\mathbb{F}_{n}$. We say that $\Phi$ is reducible if there exist proper free factors $\mathbb{F}_{n_{1}}, \mathbb{F}_{n_{2}}, \ldots, \mathbb{F}_{n_{k}}$ of $\mathbb{F}_{n}$ whose conjugacy classes are permuted under $\Phi$ and such that $\mathbb{F}_{n_{1}} * \mathbb{F}_{n_{2}} * \cdots * \mathbb{F}_{n_{k}}$ is a free factor of $\mathbb{F}_{n}$. Another formulation of the notion of reducibility is that there exists a topological representative for $\Phi$ that admits a proper invariant subgraph 
whose fundamental group is non trivial. If an endomorphism is not reducible then we say that it is irreducible.

A topological representative $f: G \longrightarrow G$ for $\Phi$ will be said to be efficient if it has no invariant forests, $G$ has no valence-one vertices, and if for all $k \in \mathbb{N}$, the restriction of $f^{k}$ to the interior of each edge of $G$ is locally injective. Also, a graph map $f: G \longrightarrow G$ will be called expanding if $G$ is equipped with a metric such that $f$ linearly expands each edge $e$ by a factor $\lambda(e)>1$.

The minimality of the dynamics of efficient representatives is asserted by the following theorem.

Theorem B. Let $f: G \longrightarrow G$ be an efficient, expanding representative of an irreducible endomorphism $\Phi$ of a free group of rank $n$. Then there exists a cofinite subset $\mathcal{B}$ of the set of periodic orbits of $f$ with the property that, for each representative $g: G^{\prime} \longrightarrow G^{\prime}$ of $\Phi$, there exists a pattern-preserving injective map from $\mathcal{B}$ to the set of periodic orbits of $g$. Moreover, the number of periodic points of $f$ whose orbit does not belong to $\mathcal{B}$ is at most $10(n-1)$.

It follows from the proof of this theorem that a periodic orbit $P \notin \mathcal{B}$ is either an inessential periodic orbit of vertices, or else its pattern is reducible and $g$ exhibits either the pattern $[G, P, f]$ or one of its reductions. Further, each point whose orbit belongs to $\mathcal{B}$ is alone in its Nielsen class for all iterates of $f$.

A direct consequence of this theorem is that two efficient, expanding representatives of an irreducible endomorphism of a free group of rank $n$ have (with at most $20(n-1)$ exceptions) the same number of periodic orbits of any pattern.

Our theorem holds in a more general setting, the essential hypothesis being the existence of efficient representatives for the given free group endomorphism. In the irreducible case, this existence is guaranteed.

This paper is organised as follows. In Section 2 we fix our notation, and we define the notions that will be used throughout the paper. In particular, we state and prove the results for relative homotopies of pointed graphs, groupoids and their morphisms, and we define our notion of pattern. We also give an algebraic characterisation of this notion in terms of conjugacy of morphisms of the associated groupoids. In Section 3 we introduce and study the notion of reducibility of patterns of periodic orbits. In Section 4 we deal with the persistence of patterns under homotopy equivalence, and prove Theorem A. Finally, in Section 5 we study the index of points and fixed point classes of expanding efficient representatives, and we prove Theorem B.

\section{General Definitions}

In this paper, all graphs will be finite. As usual, a graph will be considered to be a compact topological space, as well as a combinatorial object given by a finite set of vertices and edges. Recall that the fundamental group of a graph $G$ is free; its rank will be denoted by $|G|$.

Given a point $x$ of a graph $G$, let $d(x)$ denote its valence. Any point of valence different from 2 will be called a vertex, and the set of vertices of $G$ will be denoted by $\mathrm{V}(G)$.

If $G, G^{\prime}$ are graphs, let $\mathcal{C}\left(G, G^{\prime}\right)$ denote the class of continuous maps from $G$ to $G^{\prime}$.

2.1. Homotopies of pointed graphs. A pointed graph will be a pair $(G, P)$, where $G$ is a graph and $P$ is a finite (possibly empty) subset of $G$. Given a pointed graph $(G, P)$, let $|P|$ denote the cardinality of $P$. Given pointed graphs $(G, P)$ and $\left(G^{\prime}, Q\right)$, we say that $f: G \longrightarrow G^{\prime}$ is a pointed graph map, written $f:(G, P) \longrightarrow\left(G^{\prime}, Q\right)$, if $f \in \mathcal{C}\left(G, G^{\prime}\right)$ and $f(P) \subset Q$. 


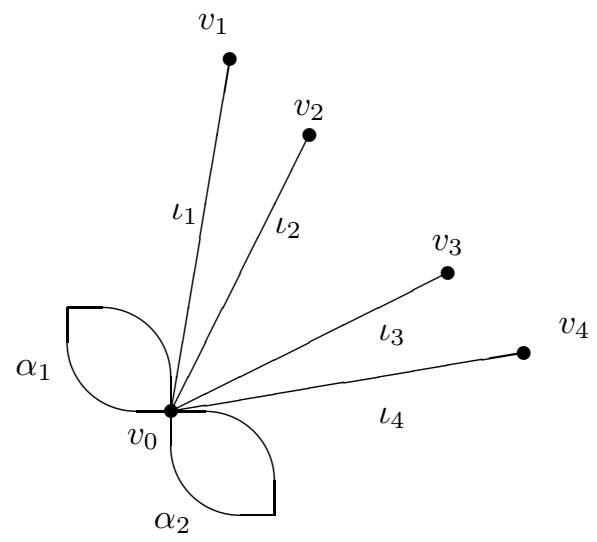

Figure 1. The pointed graph $\left(G_{2}, A_{5}\right)$

Let $(G, P)$ and $\left(G^{\prime}, Q\right)$ be pointed graphs and let $f, g:(G, P) \longrightarrow\left(G^{\prime}, Q\right)$ be pointed graph maps. We say that $f$ is homotopic to $g$ relative to $P$, written $f \simeq_{P} g$, if there exists a homotopy of pointed graph maps $\left\{h_{t}\right\}_{t \in[0,1]}:(G, P) \longrightarrow\left(G^{\prime}, Q\right)$, that is, a continuous family of pointed graph maps $h_{t}:(G, P) \longrightarrow\left(G^{\prime}, Q\right)$ with parameter $t$ ranging over $[0,1]$, and satisfying $h_{0}=f$ and $h_{1}=g$. In particular, $\left.f\right|_{P}=\left.h_{t}\right|_{P}=\left.g\right|_{P}$ for all $t \in[0,1]$. We will also write $f \simeq_{P} g$ if we do not need to specify the homotopy. If $P=Q=\emptyset$ then we obtain the usual homotopy relation between graph maps, and in this case we will simply drop all of the ' $P$ ' subscripts in the notation.

The pointed graphs $(G, P)$ and $\left(G^{\prime}, Q\right)$ will be said to have the same homotopy type, written $(G, P) \simeq\left(G^{\prime}, Q\right)$, if there exists a homotopy equivalence between them. This means that there exist maps $r:(G, P) \longrightarrow\left(G^{\prime}, Q\right)$ and $s:\left(G^{\prime}, Q\right) \longrightarrow(G, P)$ such that $r \circ s \simeq_{Q} \operatorname{Id}_{G^{\prime}}$ and $s \circ r \simeq_{P} \operatorname{Id}_{G}$. The relation of homotopy type defines an equivalence relation on the set of pointed graphs. Notice that if $(G, P) \simeq\left(G^{\prime}, Q\right)$ then $|G|=\left|G^{\prime}\right|$ and $|P|=|Q|$. We shall see shortly (Corollary 2.2) that the converse is also true.

Let $\left(G_{k}, A_{n}\right)$ denote the pointed graph with $\mathrm{V}\left(G_{k}\right)=A_{n}=\left\{v_{0}, v_{1}, \ldots, v_{n-1}\right\}$ and whose set of $k+n-1$ edges is $\left\{\alpha_{1}, \alpha_{2}, \ldots, \alpha_{k}, \iota_{1}, \iota_{2}, \ldots, \iota_{n-1}\right\}$ such that:

(i) $v_{0}$, called the distinguished vertex, is the unique vertex incident with $\alpha_{i}$ for $i=1,2, \ldots, k$.

(ii) $v_{0}$ and $v_{i}$ are the unique vertices incident with $\iota_{i}$ for $i=1,2, \ldots, n-1$.

We call the edges $\alpha_{i}$ and $\iota_{i}$ the petals and hairs of $\left(G_{k}, A_{n}\right)$ respectively. The pointed graph $\left(G_{2}, A_{5}\right)$ is illustrated in Figure 1.

As we shall see in Proposition 2.1, each homotopy equivalence class contains a $\left(G_{k}, A_{n}\right)$ which will serve as a standard model for that class. The fact that the distinguished vertex of $G_{k}$ belongs to $A_{n}$ will enable us to choose paths between points of $A_{n}$ in a natural way, and as such, will simplify some of the proofs.

Proposition 2.1. Let $(G, P)$ be a pointed graph. If $k=|G|$ and $n=|P|$ then $(G, P) \simeq\left(G_{k}, A_{n}\right)$.

Proof. We start by adding $n$ hairs $\iota_{1}, \ldots, \iota_{n}$ to $G$, each one based at a different point of $P$. We thus obtain a new graph $H_{1}$ which contains $G$ as a proper subgraph. Clearly $H_{1}$ has a set $Q$ of $n$ new valence-one vertices. It is easily seen that $(G, P) \simeq$ $\left(H_{1}, Q\right)$.

There exists a minimal tree $T \subset G \subset H_{1}$ such that $P \cup \mathrm{V}(G) \subset T$. Let $H_{2}$ be the graph obtained from $H_{1}$ by collapsing $T$ to a point. We thus obtain a graph which has a single vertex of valence $2 k+n$ and $n$ valence-one vertices. Let $R$ denote 
the set of valence-one vertices of $H_{2}$. Since $H_{2}$ is obtained from $H_{1}$ by collapsing a tree, then $\left(H_{1}, Q\right) \simeq\left(H_{2}, R\right)$.

Lastly, let $\mathrm{H}_{3}$ be the graph obtained from $\mathrm{H}_{2}$ by collapsing one of its hairs, and let $S=V\left(H_{3}\right)$. Then $\left(H_{2}, R\right) \simeq\left(H_{3}, S\right)=\left(G_{k}, A_{n}\right)$.

Corollary 2.2. Let $(G, P)$ and $\left(G^{\prime}, Q\right)$ be pointed graphs. Then $(G, P) \simeq\left(G^{\prime}, Q\right)$ if and only if $|G|=\left|G^{\prime}\right|$ and $|P|=|Q|$.

2.2. Paths and groupoids. We are now going to associate an algebraic structure, namely that of groupoid, to each pointed graph. The reader may consult [9] for further details. We will adopt a topological viewpoint, the details of which we now make explicit for completeness.

Given a graph $G$, a path in $G$ will be a continuous map $\sigma:[0,1] \longrightarrow G$. The points $\sigma(0)$ and $\sigma(1)$ will be called the endpoints of $\sigma$. If we need to specify the beginning and the end of $\sigma$, we will speak of a path from $\sigma(0)$ to $\sigma(1)$ in $G$.

The path $\sigma(1-t)$ from $\sigma(1)$ to $\sigma(0)$, denoted by $\sigma^{-1}$, will be called the inverse of $\sigma$. A path which begins and ends at the same point will be called a loop. Given two paths $\sigma$ and $\tau$ in $G$ such that $\sigma(1)=\tau(0)$, we denote their concatenation by $\sigma \tau$.

Given a pointed graph $(G, P)$, let $\wp(G, P)$ denote the set of all paths in $G$ whose endpoints belong to $P$. If $\sigma, \tau \in \wp(G, P)$ then we say that $\sigma$ and $\tau$ are equivalent if $\sigma$ is homotopic to $\tau$, keeping endpoints fixed during the homotopy. This defines an equivalence relation on $\wp(G, P)$. Let $\pi(G, P)$ denote the resulting quotient of $\wp(G, P)$, and let $[\sigma]$ denote the equivalence class of $\sigma$. The concatenation operation on $\wp(G, P)$ induces a well-defined natural product on $\pi(G, P)$, defined by $[\sigma] \cdot[\tau]=[\sigma \tau]$, and equips it with a groupoid structure. We set $[\sigma]^{-1}=\left[\sigma^{-1}\right]$. We remark that $\pi(G, P)$ has $n=|P|$ trivial elements which are the classes of the trivial loops based at the points of $P$.

Let $\mathcal{P}$ be a finite subset of $\pi(G, P)$ each of whose elements may be represented by a path which is not a loop. A finite product $\sigma_{1} \cdot \sigma_{2} \cdots \sigma_{m}$ will be called $\mathcal{P}$-admissible if either $\sigma_{i} \in \mathcal{P}$ or $\sigma_{i}^{-1} \in \mathcal{P}$, and $\sigma_{i} \neq \sigma_{i+1}^{-1}$ for all $i$. We will say that $\mathcal{P}$ is independent if any $\mathcal{P}$-admissible product is the class of a path which is not a loop. Clearly any subset of an independent set is also independent. The set $\mathcal{P}$ will be called transitive if for any pair of distinct points $x, y \in P$, there is a path $\sigma \in \wp(G, P)$ from $x$ to $y$ such that $[\sigma]$ is $\mathcal{P}$-admissible. If $\mathcal{P}$ is independent and transitive then it will be called a free system of path generators.

Proposition 2.3. Let $\mathcal{P}$ be a subset of $\pi(G, P)$ of cardinality $r$. The following assertions hold:

(a) If $\mathcal{P}$ is an independent set then $r \leq|P|-1$.

(b) $\mathcal{P}$ is a free system of path generators if and only if $\mathcal{P}$ is an independent set and $r=|P|-1$.

(c) $\mathcal{P}$ is a free system of path generators if and only if $\mathcal{P}$ is a transitive set and $r=|P|-1$.

Furthermore, $\pi(G, P)$ admits a free system of path generators.

Proof. Set $P=\left\{x_{1}, \ldots, x_{n}\right\}$, and define the graph $\mathrm{G}_{\mathcal{P}}$ as follows: its set of vertices is of cardinality $n$, say $\left\{v_{1}, \ldots, v_{n}\right\}$, and it has an edge incident at $v_{j}$ and $v_{k}$ if there exists a path $\sigma \in \wp(G, P)$ from $x_{j}$ to $x_{k}$ such that $[\sigma] \in \mathcal{P}$. The properties of $\mathcal{P}$ may be expressed in terms of properties of $\mathrm{G}_{\mathcal{P}}$. In particular:

(i) $r=\alpha$ and $n=\nu$, where $\alpha$ and $\nu$ are respectively the cardinal of the sets of edges and vertices of $\mathrm{G}_{\mathcal{P}}$.

(ii) $\mathcal{P}$ is an independent set if and only if $G_{\mathcal{P}}$ is a disjoint union of trees. 
(iii) $\mathcal{P}$ is a transitive set if and only if $G_{\mathcal{P}}$ is a connected graph.

(iv) $\mathcal{P}$ is a free system of path generators if and only if $G_{\mathcal{P}}$ is a tree.

From the well-known characterisation of trees, we have that the following properties are equivalent:

(1) $G_{\mathcal{P}}$ is a tree.

(2) $\mathrm{G}_{\mathcal{P}}$ is a disjoint union of trees and $\alpha=\nu-1$.

(3) $\mathrm{G}_{\mathcal{P}}$ is a connected graph and $\alpha=\nu-1$.

Furthermore, if $\mathrm{G}_{\mathcal{P}}$ is a disjoint union of trees then $\alpha \leq \nu-1$. Thus (a), (b) and (c) follow.

In order to obtain a free system of path generators, it suffices to take any set $\mathcal{P}$ of cardinality $n$ for which $\mathrm{G}_{\mathcal{P}}$ is a tree; this is always possible.

Let $(G, P)$ be a marked graph, with $k=|G|$ and take $x_{0} \in P$. We will identify $\pi\left(G,\left\{x_{0}\right\}\right)$ with the free group of rank $k$. Let $\left\{\theta_{1}, \ldots, \theta_{k}\right\}$ be a free basis of $\pi\left(G,\left\{x_{0}\right\}\right)$, and let us choose a free system of path generators $\left\{\left[\sigma_{1}\right], \ldots,\left[\sigma_{n-1}\right]\right\}$ of $\pi(G, P)$. Then any element of $\pi(G, P)$ may be expressed uniquely (without cancellation) as a product of $\left[\sigma_{i}\right]$ 's and $\theta_{j}$ 's. The set $\left\{\theta_{1}, \ldots, \theta_{k},\left[\sigma_{1}\right], \ldots,\left[\sigma_{n-1}\right]\right\}$ will be called a free system of generators of $\pi(G, P)$. Any groupoid morphism is determined by its effect on a free system of generators. Moreover, a morphism $\phi: \pi(G, P) \longrightarrow \pi\left(G^{\prime}, P^{\prime}\right)$ is an isomorphism if and only if it induces a bijective map from a free system of generators of $\pi(G, P)$ to a free system of generators of $\pi\left(G^{\prime}, P^{\prime}\right)$

Lemma 2.4. Let $(G, P)$ and $(G, Q)$ be pointed graphs, and let $P=\left\{x_{0}, \ldots, x_{n-1}\right\}$, $Q=\left\{y_{0}, \ldots, y_{n-1}\right\}$ and $d=\left\{d_{0}, \ldots, d_{n-1}\right\}$, where for each $i \in\{0, \ldots, n-1\}, d_{i}$ is a path from $y_{i}$ to $x_{i}$. Then the morphism $\phi_{d}: \pi(G, P) \longrightarrow \pi(G, Q)$, defined by $\phi_{d}(\alpha)=\left[d_{i}\right] \cdot \alpha \cdot\left[d_{j}\right]^{-1}$ for each $i, j \in\{o, 1, \ldots, n-1\}$ and each class $\alpha$ of paths from $x_{i}$ to $x_{j}$, is an isomorphism.

Proof. By taking a system of generators $\left\{\theta_{1}, \ldots, \theta_{k}\right\}$ of $\pi\left(G,\left\{x_{0}\right\}\right)$ and a free system of path generators $\left\{\left[\sigma_{1}\right], \ldots,\left[\sigma_{n-1}\right]\right\}$, where $\sigma_{i}$ is a path between $x_{0}$ and $x_{i}$, we obtain a free system of generators of $\pi(G, P)$. By Proposition 2.3, the following set:

$$
\left\{\phi_{d}\left(\theta_{1}\right), \ldots, \phi_{d}\left(\theta_{k}\right), \phi_{d}\left(\left[\sigma_{1}\right]\right), \ldots, \phi_{d}\left(\left[\sigma_{n-1}\right]\right)\right\}
$$

is also a free system of generators of $\pi(G, Q)$.

A pointed graph map $f:(G, P) \longrightarrow\left(G^{\prime}, Q\right)$ induces a groupoid morphism:

$$
f^{*}: \pi(G, P) \longrightarrow \pi\left(G^{\prime}, Q\right),
$$

defined by $f^{*}([\sigma])=[f \circ \sigma]$ for all $\sigma \in \wp(G, P)$. Since each groupoid morphism sends trivial elements to trivial elements, any morphism $\phi: \pi(G, P) \longrightarrow \pi\left(G^{\prime}, Q\right)$ induces a unique map from $P$ to $Q$ denoted by $\phi_{P}$. Clearly, if $\phi=f^{*}$ then $\phi_{P}=\left.f\right|_{P}$.

Note that for general maps between two spaces, the definition of an induced morphism of either the fundamental group or groupoid requires a choice of arbitrary paths between the base points and their images. In our situation, base points are mapped to base points. Our definition of the induced morphism is that obtained by choosing these paths to be trivial.

The next lemma follows easily.

Lemma 2.5. Let $f:(G, P) \longrightarrow\left(G^{\prime}, Q\right)$ and $g:\left(G^{\prime}, Q\right) \longrightarrow\left(G^{\prime \prime}, R\right)$ be pointed graph maps. Then the following assertions hold.

(a) $(g \circ f)^{*}=g^{*} \circ f^{*}$.

(b) $\left(\operatorname{Id}_{G}\right)^{*}=\operatorname{Id}_{\pi(G, P)}$. 
(c) Assume that $f$ is a homotopy equivalence between pointed graphs, and suppose that there exists a map $\varphi:\left(G^{\prime}, Q\right) \longrightarrow(G, P)$ satisfying $f \circ \varphi \simeq_{Q} \operatorname{Id}_{G^{\prime}}$ and $\varphi \circ f \simeq_{P} \operatorname{Id}_{G}$. Then $f^{*}$ and $\varphi^{*}$ are isomorphisms, and $\left(f^{*}\right)^{-1}=\varphi^{*}$.

If $r:(G, P) \longrightarrow\left(G^{\prime}, Q\right)$ is a homotopy equivalence between pointed graphs then in particular, it is a homotopy equivalence of the graphs $G$ and $G^{\prime}$, and so the morphism $r^{*}: \pi(G,\{x\}) \longrightarrow \pi\left(G^{\prime},\{r(x)\}\right)$ is an isomorphism for each $x \in G$.

Lemma 2.6. Let $f, g:(G, P) \longrightarrow\left(G^{\prime}, Q\right)$ be pointed graph maps such that $g$ maps $P$ onto $Q$ bijectively, and for which there exists a homotopy $\left\{h_{t}\right\}_{t=0}^{1}: f \simeq g$. Let $P=\left\{x_{0}, \ldots, x_{n-1}\right\}$, and let $d=\left\{d_{0}, \ldots, d_{n-1}\right\}$, where for each $i=0, \ldots, n-1, d_{i}$ is the path defined by $d_{i}(t)=h_{t}\left(x_{i}\right)$ for all $0 \leq t \leq 1$. Then the following diagram commutes:

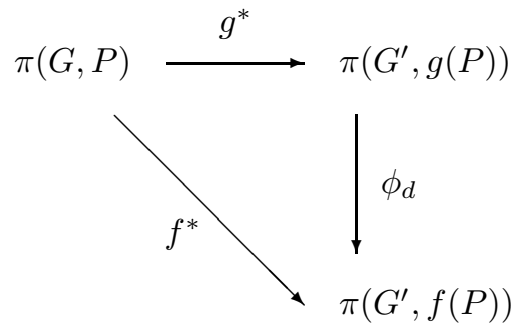

where $\phi_{d}$ is the morphism given by Lemma 2.4. Moreover, if $|g(P)|=|f(P)|$ then $\phi_{d}$ is an isomorphism.

Proof. Let $i, j \in\{0,1, \ldots, n-1\}$, and let $\beta$ be a path from $x_{i}$ to $x_{j}$. We must show that $[f \circ \beta]=\left[d_{i}\right] \cdot[g \circ \beta] \cdot\left[d_{j}\right]^{-1}=\left[d_{i}(g \circ \beta) d_{j}^{-1}\right]$, which is equivalent to showing that the paths $f \circ \beta$ and $d_{i}(g \circ \beta) d_{j}^{-1}$ are homotopic with their endpoints $f\left(x_{i}\right)$ and $f\left(x_{j}\right)$ fixed.

Let $H:[0,1] \times[0,1] \longrightarrow G$ be the map defined by:

$$
H(t, s)= \begin{cases}d_{i}(3 t) & \text { if } 0 \leq t \leq \frac{s}{3} \\ h_{s}\left(\beta\left(\frac{3 t-s}{3-2 s}\right)\right) & \text { if } \frac{s}{3} \leq t \leq \frac{3-s}{3} \\ d_{j}^{-1}(3 t-2) & \text { if } \frac{3-s}{3} \leq t \leq 1\end{cases}
$$

Then we obtain:

$$
\begin{aligned}
& \{H(t, 0)\}_{t \in[0,1]}=h_{0} \circ \beta=f \circ \beta, \\
& \{H(t, 1)\}_{t \in[0,1]}=d_{i}\left(h_{1} \circ \beta\right) d_{j}^{-1}=d_{i}(g \circ \beta) d_{j}^{-1},
\end{aligned}
$$

and

$$
\begin{aligned}
& H(0, s)=d_{i}(0)=h_{0}\left(x_{i}\right)=f\left(x_{i}\right), \\
& H(1, s)=d_{j}^{-1}(1)=h_{0}\left(x_{j}\right)=f\left(x_{j}\right),
\end{aligned}
$$

for each $s \in[0,1]$. The result follows from Lemma 2.4 .

Remark 2.7. From Lemmas 2.5 and 2.6, we see that if $r:(G, P) \longrightarrow\left(G^{\prime}, r(P)\right)$ is a homotopy equivalence (not necessarily of pointed graphs) and if $|P|=|r(P)|$ then $r^{*}$ is an isomorphism.

Proposition 2.8. Let $f, g:(G, P) \longrightarrow\left(G^{\prime}, Q\right)$ be pointed graph maps. Then $f \simeq_{P}$ $g$ if and only if $f^{*}=g^{*}$.

Proof. The proof is a routine check, that we provide for completeness. If $f \simeq_{P} g$ then by Lemma 2.6 we get $f^{*}=\phi_{d} \circ g^{*}$, where $\phi_{d}=\mathrm{Id}$. 
Conversely, suppose that $f^{*}=g^{*}$, and set $|G|=k,\left|G^{\prime}\right|=l,|P|=n$ and $|Q|=m$. From Proposition 2.1 there exist pointed graph maps:

$$
\begin{array}{ll}
r_{1}:(G, P) \longrightarrow\left(G_{k}, A_{n}\right) & s_{1}:\left(G_{k}, A_{n}\right) \longrightarrow(G, P) \\
r_{2}:\left(G^{\prime}, Q\right) \longrightarrow\left(G_{l}, A_{m}\right) & s_{2}:\left(G_{l}, A_{m}\right) \longrightarrow\left(G^{\prime}, Q\right)
\end{array}
$$

such that $\left(r_{1} \circ s_{1}\right) \simeq_{A_{n}} \operatorname{Id}_{G_{k}},\left(s_{1} \circ r_{1}\right) \simeq_{P} \operatorname{Id}_{G},\left(r_{2} \circ s_{2}\right) \simeq_{A_{m}} \operatorname{Id}_{G_{l}}$ and $\left(s_{2} \circ r_{2}\right) \simeq_{Q}$ $\operatorname{Id}_{G^{\prime}}$.

Set $P=\left\{p_{0}, \ldots, p_{n-1}\right\}, Q=\left\{q_{0}, \ldots, q_{m-1}\right\}, A_{n}=\left\{v_{0}, \ldots, v_{n-1}\right\}$, and $A_{m}=$ $\left\{w_{0}, \ldots, w_{m-1}\right\}$. By re-indexing if necessary, we may suppose that $f\left(p_{0}\right)=g\left(p_{0}\right)=$ $q_{0}, r_{1}\left(p_{0}\right)=v_{0}$, and $r_{2}\left(q_{0}\right)=w_{0}$. Consider the two maps:

$$
r_{2} \circ f \circ s_{1} \text { and } r_{2} \circ g \circ s_{1}:\left(G_{k}, A_{n}\right) \longrightarrow\left(G_{l}, A_{m}\right) \text {. }
$$

Notice that $\left(r_{2} \circ f \circ s_{1}\right)\left(v_{0}\right)=w_{0}=\left(r_{2} \circ g \circ s_{1}\right)\left(v_{0}\right)$. It follows from the hypothesis and Lemma 2.5 that $\left(r_{2} \circ f \circ s_{1}\right)^{*}=\left(r_{2} \circ g \circ s_{1}\right)^{*}$.

We claim that $\left(r_{2} \circ f \circ s_{1}\right) \simeq_{A_{n}}\left(r_{2} \circ g \circ s_{1}\right)$. Indeed, let $\alpha_{1}, \ldots, \alpha_{k}$ and $\iota_{1}, \ldots, \iota_{n-1}$ be the petals and the hairs of $\left(G_{k}, A_{n}\right)$. Then:

$$
\begin{array}{ll}
\left(r_{2} \circ f \circ s_{1}\right)\left(\alpha_{i}\right) \simeq_{\left\{v_{0}\right\}}\left(r_{2} \circ g \circ s_{1}\right)\left(\alpha_{i}\right) & \text { for } i=1, \ldots, k \\
\left(r_{2} \circ f \circ s_{1}\right)\left(\iota_{i}\right) \simeq_{\left\{v_{0}, v_{i}\right\}}\left(r_{2} \circ g \circ s_{1}\right)\left(\iota_{i}\right) & \text { for } i=1, \ldots, n-1 .
\end{array}
$$

For $i=1, \ldots, k$ and $t \in[0,1]$, let $h_{t}^{i}(x):\left(\alpha_{i}, v_{0}\right) \longrightarrow\left(G_{l}, A_{m}\right)$ be a homotopy between $\left.\left(r_{2} \circ f \circ s_{1}\right)\right|_{\alpha_{i}}$ and $\left.\left(r_{2} \circ g \circ s_{1}\right)\right|_{\alpha_{i}}$ relative to $v_{0}$. Similarly, for $i=$ $1, \ldots, n-1$ and $t \in[0,1]$, let $l_{t}^{i}(x):\left(\iota_{i},\left\{v_{0}, v_{i}\right\}\right) \longrightarrow\left(G_{l}, A_{m}\right)$ be a homotopy between $\left.\left(r_{2} \circ f \circ s_{1}\right)\right|_{\iota_{i}}$ and $\left.\left(r_{2} \circ g \circ s_{1}\right)\right|_{\iota_{i}}$ relative to $v_{0}$ and $v_{i}$. For $t \in[0,1]$, define $H_{t}:\left(G_{k}, A_{n}\right) \longrightarrow\left(G_{l}, A_{m}\right)$ in the following way:

$$
H_{t}(x)= \begin{cases}h_{t}^{i}(x) & \text { if } x \in \alpha_{i}, \\ l_{t}^{i}(x) & \text { if } x \in \iota_{i} .\end{cases}
$$

Since for all $i, j$ and $t$, the unique common points of the $\alpha_{i}$ 's and $\iota_{j}$ 's are $v_{0}$, and $h_{t}^{i}\left(v_{0}\right)=l_{t}^{j}\left(v_{0}\right)=w_{0}$, the map $H_{t}$ is well defined and is a homotopy between $r_{2} \circ f \circ s_{1}$ and $r_{2} \circ g \circ s_{1}$ relative to $A_{n}$. Thus $r_{2} \circ f \circ s_{1} \simeq_{A_{n}} r_{2} \circ g \circ s_{1}$, which proves the claim.

Therefore $s_{2} \circ r_{2} \circ f \circ s_{1} \circ r_{1} \simeq_{P} s_{2} \circ r_{2} \circ g \circ s_{1} \circ r_{1}$. Thus $f \simeq_{P} g$, and this completes the proof.

The following proposition asserts the existence of topological representatives for groupoid morphisms.

Proposition 2.9. Let $\psi: \pi(G, P) \longrightarrow \pi\left(G^{\prime}, Q\right)$ be a groupoid morphism. Then there exists a pointed graph map $r:(G, P) \longrightarrow\left(G^{\prime}, Q\right)$ such that $r^{*}=\psi$. Moreover, $\psi$ is an isomorphism if and only if $r$ is a homotopy equivalence between pointed graphs.

Proof. In the special case where both pointed graphs are standard models (of the form $\left(G_{k}, A_{n}\right)$ ), the first statement is clear, and the second statement follows from Proposition 2.8. The general case may be reduced to this special case, as in the proof of Proposition 2.8, by using homotopy equivalences which send each pointed graph to the corresponding standard model.

2.3. Patterns. Let $G$ and $G^{\prime}$ be graphs, $f \in \mathcal{C}(G, G)$ and $f^{\prime} \in \mathcal{C}\left(G^{\prime}, G^{\prime}\right)$. The pairs $(G, f)$ and $\left(G^{\prime}, f^{\prime}\right)$ will be said to be equivalent, written $(G, f) \sim\left(G^{\prime}, f^{\prime}\right)$, if there exists a homotopy equivalence $r: G \longrightarrow G^{\prime}$ with the property that $r \circ f \simeq g \circ r$. Then $(G, f) \sim\left(G^{\prime}, f^{\prime}\right)$ if and only if $f$ and $f^{\prime}$ are representatives of the same endomorphism of a free group of finite rank. 
Let $\Sigma$ denote the set of triples $(G, P, f)$, where $(G, P)$ is a pointed graph and $f:(G, P) \longrightarrow(G, P)$ is a pointed graph map. Two elements $(G, P, f),\left(G^{\prime}, Q, g\right) \in \Sigma$ will be said to have the same pattern, written $(G, P, f) \sim\left(G^{\prime}, Q, g\right)$, if there exists a homotopy equivalence $r:(G, P) \longrightarrow\left(G^{\prime}, Q\right)$ between pointed graphs such that the following diagram:

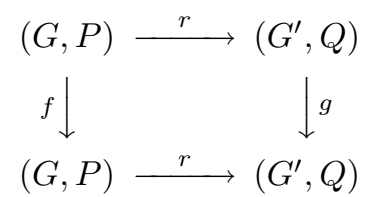

commutes up to homotopy relative to $P$. In other words, $g \circ r \simeq_{P} r \circ f$. This defines an equivalence relation on $\Sigma$. The resulting equivalence class, or pattern, of $(G, P, f)$ will be denoted by $[G, P, f]$. If $(G, P, f),\left(G^{\prime}, Q, g\right)$ have the same pattern then it follows from Corollary 2.2 that $|G|=\left|G^{\prime}\right|$ and $|P|=|Q|$. As we shall see, the condition in the definition of pattern that $r$ be a homotopy equivalence between pointed graphs may be relaxed. Indeed, by Corollary 2.12 it will suffice to take any homotopy equivalence between $G$ and $G^{\prime}$ which induces a bijection between the marked points.

Remark 2.10. This notion of pattern in some sense unifies the previous notions in the literature. In order to recover the different specific notions of pattern, it suffices to specify the hypotheses on the map $r$ in such a way that the desired properties are preserved. In our framework, the homotopy type of the space will be preserved and the hypothesis is just that $r$ be a homotopy equivalence. If one wants to preserve the space itself, $r$ must be a homeomorphism, as in the case of the interval [19], of fixed graphs [3] or of surfaces [8, 16, 18]. For patterns of trees (see [1]), one wants to preserve the "relative positions" of the points of the orbit and this is the condition that must be satisfied by $r$.

The following result is an algebraic characterisation of the notion of pattern, and gives us a powerful tool for deciding when two elements of $\Sigma$ have the same pattern. More precisely, two patterns coincide if and only if the induced groupoid morphisms are conjugate. A pattern may thus be thought of as a conjugacy class of groupoid endomorphisms.

Theorem 2.11. Let $(G, P, f),\left(G^{\prime}, Q, g\right) \in \Sigma$. Then $(G, P, f)$ and $\left(G^{\prime}, Q, g\right)$ have the same pattern if and only if there exists an isomorphism $\phi: \pi(G, P) \longrightarrow \pi\left(G^{\prime}, Q\right)$ such that $g^{*} \circ \phi=\phi \circ f^{*}$.

Proof. Suppose that $(G, P, f) \sim\left(G^{\prime}, Q, g\right)$, and let $r:(G, P) \longrightarrow\left(G^{\prime}, Q\right)$ be the corresponding homotopy equivalence of pointed graphs satisfying $g \circ r \simeq_{P} r \circ f$. It follows from Lemma 2.5 that $r^{*}$ is an isomorphism, and that $g^{*} \circ r^{*}=(g \circ r)^{*}=$ $(r \circ f)^{*}=r^{*} \circ f^{*}$.

Conversely, suppose that there exists an isomorphism $\phi: \pi(G, P) \longrightarrow \pi\left(G^{\prime}, Q\right)$ such that $g^{*} \circ \phi=\phi \circ f^{*}$. It follows from Proposition 2.9 that there exists a homotopy equivalence $r:(G, P) \longrightarrow\left(G^{\prime}, Q\right)$ of pointed graphs such that $\phi=r^{*}$. Then $g^{*} \circ r^{*}=r^{*} \circ f^{*}$, and by Proposition 2.8 we obtain the desired homotopy equivalence $g \circ r \simeq_{P} r \circ f$.

Corollary 2.12. Let $(G, P, f),\left(G^{\prime}, Q, g\right) \in \Sigma$. Then $(G, P, f)$ and $\left(G^{\prime}, Q, g\right)$ have the same pattern if and only if there exists a homotopy equivalence $r: G \longrightarrow G^{\prime}$ such that $r$ maps $P$ bijectively onto $Q$, and the following diagram:

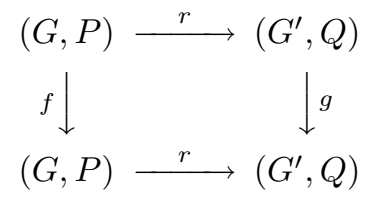


commutes up to homotopy relative to $P$, i.e., $r \circ f \simeq_{P} g \circ r$.

Proof. If $[G, P, f]=\left[G^{\prime}, Q, g\right]$ then such a map $r$ exists by definition.

Conversely, if $r: G \longrightarrow G^{\prime}$ is a homotopy equivalence between $G$ and $G^{\prime}$ such that $r$ maps $P$ bijectively onto $Q$ then it follows from Remark 2.7 that the induced morphism $r^{*}: \pi(G, P) \longrightarrow \pi\left(G^{\prime}, Q\right)$ is an isomorphism. Thus $g^{*} \circ r^{*}=r^{*} \circ f^{*}$, and the result follows by Theorem 2.11 .

Remark 2.13. Theorem 2.11 highlights the equivalence between the problem of deciding whether two elements of $\Sigma$ define the same pattern and that of deciding whether two groupoid endomorphisms are conjugate. This is a difficult question. For instance, if $|G|=k$ and $|P|=1$, this comes down to deciding whether two endomorphisms of the free group of rank $k$ are conjugate. This problem was solved for a particular class (irreducible) of $\operatorname{Out}\left(\mathbb{F}_{k}\right)[15,21]$, and in general for $\operatorname{Aut}\left(\mathbb{F}_{k}\right)$ and $\operatorname{Out}\left(\mathbb{F}_{k}\right)$ [17]. The general cases of free group and free groupoid endomorphisms are still open, and are interesting in their own right. They may however be answered in certain special cases, as we will see shortly in Examples 2.15 and 2.16.

We are now going to analyse some basic properties of the relation "to have the same pattern" in the case of periodic orbits. Let $(G, P, f),\left(G^{\prime}, P^{\prime}, f^{\prime}\right) \in \Sigma$ be such that $(G, f) \sim\left(G^{\prime}, f^{\prime}\right)$, and $P$ and $P^{\prime}$ are periodic orbits of $f$ and $f^{\prime}$ respectively. If the fundamental groups of $G$ and $G^{\prime}$ are trivial then it follows from Theorem 2.11 that the pattern is characterised by $|P|$. This is not the case if the fundamental groups are non trivial, even for the circle (see Example 2.14). Another apparently simple situation is that of patterns of fixed points. From Theorem 2.11, all fixed points of circle maps of the same degree have the same pattern. Example 2.15 shows that this is not true for (slightly) more complicated graphs.

If $P$ is a periodic orbit of period $|P|>1$ and $m \in \mathbb{N}$ is a divisor of $|P|$ then $P$ contains different subsets which are periodic orbits of $f^{m}$. In this context, it is natural to consider the following related question: let $x, y$ be distinct points of $P$. Then $\operatorname{Orb}_{f^{m}}(x)$ and $\operatorname{Orb}_{f^{m}}(y)$ are periodic orbits of $f^{m}$. Is it true that $\left[G, \operatorname{Orb}_{f m}(x), f^{m}\right]=\left[G, \operatorname{Orb}_{f m}(y), f^{m}\right]$ ? If $f$ is a homotopy equivalence then the answer is positive. However, the answer to this question in general is negative. Indeed, we show in Example 2.16 that two points of a periodic orbit of period 2 considered as fixed points of $f^{2}$ may have different patterns.

Example 2.14 (Two periodic orbits with the same period may have different pattern). Let $\mathbb{S}^{1}$ be the circle, and let $\alpha$ and $\beta$ be injective paths in $\mathbb{S}^{1}$ such that $\alpha(1)=\beta(0), \beta(1)=\alpha(0)$, and $\alpha((0,1)) \cap \beta((0,1))=\emptyset$. Consider two maps $f, g \in \mathcal{C}\left(\mathbb{S}^{1}, \mathbb{S}^{1}\right)$ defined by:

$$
\left\{\begin{array}{l}
f \circ \alpha=\alpha^{-1} \\
f \circ \beta=\alpha \beta \alpha
\end{array}, \quad\left\{\begin{array}{c}
g \circ \alpha=\beta \\
g \circ \beta=\alpha .
\end{array}\right.\right.
$$

For both of these maps, the set $P=\{\alpha(0), \alpha(1)\}$ is a periodic orbit of period 2 but $\left[\mathbb{S}^{1}, P, f\right] \neq\left[\mathbb{S}^{1}, P, g\right]$. To see this, we will apply Theorem 2.11. Consider a free system of generators $\{a, b\}$, where $a=[\alpha]$ and $b=[\alpha \beta]$. The induced maps on $\pi\left(\mathbb{S}^{1}, P\right)$ satisfy:

$$
\left\{\begin{array}{l}
f^{*}(a)=a^{-1} \\
f^{*}(b)=a^{-1} b a
\end{array}, \quad\left\{\begin{array}{l}
g^{*}(a)=a^{-1} b \\
g^{*}(b)=a^{-1} b a .
\end{array}\right.\right.
$$


Suppose that there exists an isomorphism $\phi: \pi\left(\mathbb{S}^{1}, P\right) \longrightarrow \pi\left(\mathbb{S}^{1}, P\right)$ such that $g^{*} \circ$ $\phi=\phi \circ f^{*}$. That is,

$$
\begin{aligned}
& g^{*}(\phi(a))=\phi\left(f^{*}(a)\right)=\phi\left(a^{-1}\right)=\phi(a)^{-1}, \text { and } \\
& g^{*}(\phi(b))=\phi\left(f^{*}(b)\right)=\phi\left(a^{-1} b a\right)=\phi(a)^{-1} \phi(b) \phi(a) .
\end{aligned}
$$

Since $\phi$ is an isomorphism, it sends trivial elements to trivial elements and loops to loops. We thus consider 4 cases (for simplicity, the classes of the trivial loops based at each point of $P$ are identified with the corresponding point of $P$ ):

(i) $\phi(\alpha(0))=\alpha(0)$ and $\phi(b)=b$ (thus $\phi(a)=b^{n} a$ with $n \in \mathbb{Z}$ ),

(ii) $\phi(\alpha(0))=\alpha(0)$ and $\phi(b)=b^{-1}$ (thus $\phi(a)=\left(b^{-1}\right)^{n} a=b^{-n} a$ with $n \in \mathbb{Z}$ ),

(iii) $\phi(\alpha(0))=\alpha(1)$ and $\phi(b)=a^{-1} b a=[\beta \alpha]\left(\right.$ thus $\phi(a)=\left(a^{-1} b a\right)^{n} a^{-1}=a^{-1} b^{n}$ with $n \in \mathbb{Z})$,

(iv) $\phi(\alpha(0))=\alpha(1)$ and $\phi(b)=a^{-1} b^{-1} a=[\beta \alpha]^{-1}$ (thus $\phi(a)=\left(a^{-1} b^{-1} a\right)^{n} a^{-1}=$ $a^{-1} b^{-n}$ with $\left.n \in \mathbb{Z}\right)$.

In the first case we see that:

$$
a^{-1} b^{-n}=\phi(a)^{-1}=g^{*}(\phi(a))=\left(a^{-1} b a\right)^{n} a^{-1} b=a^{-1} b^{n} a a^{-1} b=a^{-1} b^{n+1} .
$$

The only solution of this equation is $n=-1 / 2$; a contradiction. The proof follows similarly in the remaining three cases.

Example 2.15 ( Two fixed points may have different pattern). Let $G=\left(G_{3}, A_{1}\right)$, and set $P=\left\{v_{0}\right\}$. Let $f:(G, P) \longrightarrow(G, P)$ be a map with $f\left(v_{0}\right)=v_{0}$ such that the induced morphism $f^{*}: \pi(G, P) \longrightarrow \pi(G, P)$ satisfies:

$$
\begin{aligned}
& f^{*}\left(\theta_{1}\right)=\theta_{1} \cdot \theta_{3} \cdot \theta_{1}^{-1}, \\
& f^{*}\left(\theta_{2}\right)=\theta_{1}, \text { and } \\
& f^{*}\left(\theta_{3}\right)=\theta_{3}^{-1} \cdot \theta_{2} \cdot \theta_{3}
\end{aligned}
$$

for the free system of generators $\left\{\theta_{1}, \theta_{2}, \theta_{3}\right\}$ of $\pi(G, P)$ defined by $\theta_{i}=\left[\alpha_{i}\right]$ for $i=1,2,3$. Since $f^{*}\left(\theta_{3}\right)$ starts with $\theta_{3}^{-1}$, the map $f$ has another fixed point $q \in$ $\alpha_{3}$. If we denote by $\beta$ the injective path contained in the edge $\alpha_{3}$ with the same orientation, starting at $v_{0}$ and ending at $q$, then it follows that $[f \circ \beta]=\left[\alpha_{3}^{-1} \beta\right]$. We will show that $[G, P, f] \neq[G, Q, f]$, where $Q=\{q\}$.

To compute the induced morphism $f_{Q}^{*}: \pi(G, Q) \longrightarrow \pi(G, Q)$, we consider a free system of generators $\left\{\vartheta_{1}, \vartheta_{2}, \vartheta_{3}\right\}$ of $\pi(G, Q)$ defined by $\vartheta_{i}=\left[\beta^{-1} \alpha_{i} \beta\right]$ for $i=1,2,3$. Then the map $f_{Q}^{*}$ satisfies:

$$
\begin{aligned}
& f_{Q}^{*}\left(\vartheta_{1}\right)=\vartheta_{3} \cdot \vartheta_{1} \cdot \vartheta_{3} \cdot \vartheta_{1}^{-1} \cdot \vartheta_{3}^{-1}, \\
& f_{Q}^{*}\left(\vartheta_{2}\right)=\vartheta_{3} \cdot \vartheta_{1} \cdot \vartheta_{3}^{-1}, \text { and } \\
& f_{Q}^{*}\left(\vartheta_{3}\right)=\vartheta_{2} .
\end{aligned}
$$

Notice that $\delta=\theta_{1} \cdot \theta_{2} \cdot \theta_{3}$ is a fixed element of $\left.\pi(G, P)\right)$ under $f^{*}$. We claim that $f_{Q}^{*}$ has no non-trivial fixed element (meaning fixed and not fixed up to inner automorphisms). Thus the patterns $[G, P, f]$ and $[G, Q, f]$ are different. Before showing that this is the case, let us make some comments about this example. The morphisms $f^{*}$ and $f_{Q}^{*}$ are induced by a pseudo-Anosov homeomorphism $h$ of the 2 -disc $\mathbb{D}^{2}$ relative to one of its periodic orbits of period 3 . The only non-trivial free homotopy class left invariant under the action of $h$ is that of the boundary $\partial \mathbb{D}^{2}$. The fixed element $\delta$ of $f^{*}$ corresponds to the class of $\partial \mathbb{D}^{2}$ taking the basepoint to be also on $\partial \mathbb{D}^{2}$. On the other hand, $f^{*}$ and $f_{Q}^{*}$ differ essentially by an inner automorphism. Then $f_{Q}^{*}$ does not fix the element of $\pi(G, Q)$ corresponding to $\partial \mathbb{D}^{2}$, and thus it has no non-trivial fixed points. 
We now outline the steps needed to prove the claim. Suppose on the contrary that $w$ is a non-trivial reduced word in the $\vartheta_{i}$ satisfying $f_{Q}^{*}(w)=w$. First, $w$ contains at least one occurrence of $\vartheta_{3}$ or $\vartheta_{3}^{-1}$; if not, $\vartheta_{2}$ and $\vartheta_{2}^{-1}$ would not appear either in $f_{Q}^{*}(w)$, so $f_{Q}^{*}(w)=\vartheta_{1}^{l}$, where $l \neq 0$, which contradicts the fact that $f_{Q}^{*}(w)=w$. So we may write $w$ in the (reduced) form $w=w_{0} \vartheta_{3}^{k_{1}} w_{1} \vartheta_{3}^{k_{2}} \ldots w_{l-1} \vartheta_{3}^{k_{l}} w_{l}$, where the $k_{i}$ are non zero, the $w_{i}$ are reduced words in $\vartheta_{1}, \vartheta_{2}$ and their inverses, and $w_{1}, \ldots, w_{l-1}$ are non trivial. Then writing the $f_{Q}^{*}\left(w_{i}\right)$ in reduced form, $f_{Q}^{*}(w)=$ $f_{Q}^{*}\left(w_{0}\right) \vartheta_{2}^{k_{1}} f_{Q}^{*}\left(w_{1}\right) \vartheta_{2}^{k_{2}} \ldots f_{Q}^{*}\left(w_{l-1}\right) \vartheta_{2}^{k_{l}} f_{Q}^{*}\left(w_{l}\right)$ is also reduced. It follows that $w_{0}$ must be a non-trivial word containing at least one occurrence of $\vartheta_{2}$ and $\vartheta_{2}^{-1}$. From the form of $f_{Q}^{*}$, we see that $f_{Q}^{*}\left(w_{0}\left(\vartheta_{1}, \vartheta_{2}\right)\right)=\vartheta_{3} \vartheta_{1} \cdot w_{0}\left(\vartheta_{3}, \vartheta_{1}\right) \cdot \vartheta_{1}^{-1} \vartheta_{3}^{-1}$ (not necessarily reduced). Comparing the beginning of $w$ with that of $f_{Q}^{*}(w)$, and using the fact that $f_{Q}^{*}$ is an automorphism, we see that $f_{Q}^{*}(w)$ contains at least one occurrence of $\vartheta_{3}$ or $\vartheta_{3}^{-1}$. Finally, $w_{0}$ contains one of the symbols $\vartheta_{2}$ or $\vartheta_{2}^{-1}$, but neither $\vartheta_{3}$ nor $\vartheta_{3}^{-1}$, so the first occurrence of $\vartheta_{2}$ or $\vartheta_{2}^{-1}$ in $w$ comes before the first occurrence of $\vartheta_{3}$ or $\vartheta_{3}^{-1}$ in $w$. But $f_{Q}^{*}\left(w_{0}\right)$ contains one of the symbols $\vartheta_{3}$ or $\vartheta_{3}^{-1}$ but neither $\vartheta_{2}$ nor $\vartheta_{2}^{-1}$, so the first occurrence of $\vartheta_{3}$ or $\vartheta_{3}^{-1}$ in $f_{Q}^{*}(w)=w$ comes before the first occurrence of $\vartheta_{2}$ or $\vartheta_{2}^{-1}$ in $w$, a contradiction.

Observe that Theorem 2.11 refers to conjugacy (here in $\operatorname{Aut}\left(F_{3}\right)$ ), and not conjugacy up to an inner automorphism. This example highlights the difference between these two concepts.

Example 2.16 (Two points of the same orbit may have different pattern). Let $f:\left(G_{2}, A_{1}\right) \longrightarrow\left(G_{2}, A_{1}\right)$ be a pointed graph map such that the induced morphism $f^{*}$ from $\pi\left(G_{2},\left\{v_{0}\right\}\right)$ to itself satisfies:

$$
\begin{aligned}
& f^{*}\left(\theta_{1}\right)=\theta_{1} \cdot \theta_{1} \cdot \theta_{1}, \\
& f^{*}\left(\theta_{2}\right)=\theta_{2},
\end{aligned}
$$

for the free system of generators $\left\{\theta_{1}, \theta_{2}\right\}$ of $\pi\left(G_{2},\left\{v_{0}\right\}\right)$ defined by $\theta_{i}=\left[\alpha_{i}\right]$ for $i=1,2$.

The map $f$ has three periodic orbits of period 2 contained in $\alpha_{1}$. Among these three orbits, let $P$ that which has a point closest to $v_{0}$ (with respect to the orientation of $\alpha_{1}$ ). We subdivide the edge $\alpha_{1}$ at the points of $P$ into three injective paths $\beta_{1}, \beta_{2}, \beta_{3}$ (their orientation induced by that of $\left.\alpha_{1}\right)$ satisfying $\beta_{i}((0,1)) \cap P=\emptyset$ for $i=1,2,3$. These paths are defined so that $v_{0}=\beta_{1}(0)=\beta_{3}(1)$ and $x_{i}:=\beta_{i}(1)=$ $\beta_{i+1}(0) \in P$ for $i=1,2$. It follows from the definition of $P$ that $f \circ \beta_{1}=\beta_{1} \beta_{2}$, $f \circ \beta_{2}=\beta_{3} \beta_{1}$, and $f \circ \beta_{3}=\beta_{2} \beta_{3} \beta_{1} \beta_{2} \beta_{3}$.

We now consider $\left(G_{2},\left\{x_{1}\right\}, f^{2}\right)$ and $\left(G_{2},\left\{x_{2}\right\}, f^{2}\right)$ and we will show that the patterns of these two triples are different.

A free system of generators of $\pi\left(G_{2},\left\{x_{1}\right\}\right)$ is given by $a_{1}=\left[\beta_{1}^{-1} \alpha_{1} \beta_{1}\right]$ and $b_{1}=$ $\left[\beta_{1}^{-1} \alpha_{2} \beta_{1}\right]$, and a free system of generators of $\pi\left(G_{2},\left\{x_{2}\right\}\right)$ is given by $a_{2}=\left[\beta_{3} \alpha_{1} \beta_{3}^{-1}\right]$ and $b_{2}=\left[\beta_{3} \alpha_{2} \beta_{3}^{-1}\right]$. For $i=1,2$, let $\phi_{i}$ denote the morphism

$$
\left(f^{2}\right)^{*}: \pi\left(G_{2},\left\{x_{i}\right\}\right) \longrightarrow \pi\left(G_{2},\left\{x_{i}\right\}\right) .
$$

A simple computation shows that:

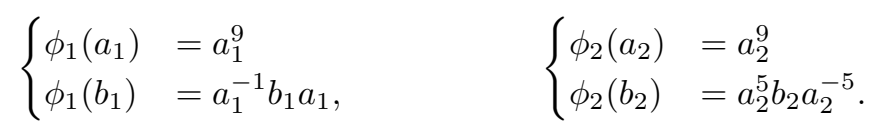

In view of Theorem 2.11 , to prove that $\left[G_{2},\left\{x_{1}\right\}, f^{2}\right] \neq\left[G_{2},\left\{x_{2}\right\}, f^{2}\right]$, one has to show that there does not exist an isomorphism $\phi: \pi\left(G_{2},\left\{x_{1}\right\}\right) \longrightarrow \pi\left(G_{2},\left\{x_{2}\right\}\right)$ such that $\phi_{2} \circ \phi=\phi \circ \phi_{1}$. Suppose on the contrary that such a $\phi$ exists. To reach 
a contradiction we can use the following simple fact: assume that $w\left(a_{2}, b_{2}\right)$ is a reduced word in $a_{2}$ and $b_{2}$. Then $w\left(a_{2}^{9}, a_{2}^{5} b_{2} a_{2}^{-5}\right)=a_{2}^{5} w\left(a_{2}^{9}, b_{2}\right) a_{2}^{-5}$.

Set $\phi\left(a_{1}\right)=w_{a}\left(a_{2}, b_{2}\right)$ and $\phi\left(b_{1}\right)=w_{b}\left(a_{2}, b_{2}\right)$, with $w_{a}\left(a_{2}, b_{2}\right)$ and $w_{b}\left(a_{2}, b_{2}\right)$ reduced words. By using $\phi\left(\phi_{1}\left(a_{1}\right)\right)=\phi_{2}\left(\phi\left(a_{1}\right)\right)$ and by studying carefully the lengths of the words appearing in these expressions, one obtains the relation $w_{a}\left(a_{2}, b_{2}\right)=$ $a_{2}^{k}$, where $k \in \mathbb{Z} \backslash\{0\}$. Moreover, since $\phi$ is an isomorphism we must have $k \in\{1,-1\}$ in order that $a_{2}$ be generated by $w_{a}\left(a_{2}, b_{2}\right)=a_{2}^{k}$ and $w_{b}\left(a_{2}, b_{2}\right)$.

We now study the images of $b_{1}$. The equality $\phi_{2}\left(\phi\left(b_{1}\right)\right)=\phi\left(\phi_{1}\left(b_{1}\right)\right)$ implies $w_{b}\left(a_{2}^{9}, a_{2}^{5} b_{2} a_{2}^{-5}\right)=a_{2}^{-k} w_{b}\left(a_{2}, b_{2}\right) a_{2}^{k}$. From this, one obtains that $w_{b}\left(a_{2}, b_{2}\right)$ begins with $a_{2}^{l} b_{2}^{m} \ldots$, with $l, m \in \mathbb{Z}, m \neq 0$. Consequently, $k=-(5+8 l) \notin\{1,-1\}$; a contradiction.

\section{REDUCIBLE AND IRREDUCIBLE PERIODIC PATTERNS}

Let $\Sigma^{\circ}$ denote the set of all $(G, P, f) \in \Sigma$ such that $P$ is a periodic orbit of $f$. Any pattern having a representative in $\Sigma^{\circ}$ will be called a periodic pattern of $f$. All representatives of such a pattern belong to $\Sigma^{\circ}$, thus the notion of periodic pattern does not depend on the choice of representative.

Given $(G, P, f),\left(G^{\prime}, Q, g\right) \in \Sigma^{\circ}$, we define the following partial order: $\left[G^{\prime}, Q, g\right] \preceq$ $[G, P, f]$ if there exists a homotopy equivalence $r: G \longrightarrow G^{\prime}$ such that $r(P)=\bar{Q}$ and the following diagram:

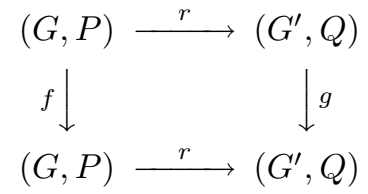

commutes up to homotopy relative to $P$. This definition does not depend on the choice of representative of the patterns. Observe also that it differs from the definition of pattern since we do not require $\left.r\right|_{P}$ to be injective here. We shall use the symbols $\prec, \succeq$ and $\succ$ in the usual way. If $\left[G^{\prime}, Q, g\right] \prec[G, P, f]$ then we shall say that $\left[G^{\prime}, Q, g\right]$ is a reduction of $[G, P, f]$. A pattern which admits a reduction will be called reducible, and irreducible otherwise.

The next two results highlight the basic properties of the relation $\preceq$.

Lemma 3.1. The following assertions hold:

(a) $\left[G^{\prime}, Q, g\right]$ is a reduction of $[G, P, f]$ if and only if $\left[G^{\prime}, Q, g\right] \preceq[G, P, f]$ and $|Q|<|P|$.

(b) Assume that $\left[G^{\prime}, Q, g\right] \preceq[G, P, f]$, and let $n=|P|$ and $m=|Q|$. Then $n=q m$ for some $q \in \mathbb{N}$.

Proof. The first statement follows directly from Corollary 2.12. For the second statement, let $r: G \longrightarrow G^{\prime}$ be a homotopy equivalence such that $r(P)=Q$ and $g \circ r \simeq_{P} r \circ f$, and let $x \in P$. Then $r(x) \in Q$, and $g^{n}(r(x))=r\left(f^{n}(x)\right)=r(x)$. Hence $m$ divides $n$.

Proposition 3.2. Suppose that $(G, P, f),\left(G^{\prime}, Q, g\right) \in \Sigma^{\circ}$ and $|Q| \leq|P|$. Then $\left[G^{\prime}, Q, g\right] \preceq[G, P, f]$ if and only if there exists $\phi: \pi(G, P) \longrightarrow \pi\left(G^{\prime}, Q\right)$, a groupoid morphism, satisfying:

(a) $g^{*} \circ \phi=\phi \circ f^{*}$.

(b) For each $x \in P, \phi: \pi(G,\{x\}) \longrightarrow \pi\left(G^{\prime},\left\{\phi_{P}(x)\right\}\right)$ is an isomorphism (of free groups), where $\phi_{P}: P \longrightarrow Q$ is the map induced by $\phi$.

Moreover, $\left[G^{\prime}, Q, g\right]=[G, P, f]$ if and only if $\phi$ is an isomorphism of groupoids. 
Proof. Suppose that $r: G \longrightarrow G^{\prime}$ is a homotopy equivalence such that $r(P)=Q$ and $g \circ r \simeq_{P} r \circ f$. Then $\phi=r^{*}$ clearly satisfies (b). Part (a) follows from Lemma 2.5(a).

Conversely, suppose that there exists $\phi$ such that (a) and (b) hold. From Proposition 2.9, there exists a pointed graph map $r:(G, P) \longrightarrow\left(G^{\prime}, Q\right)$ such that $\phi=r^{*}$. Then $g^{*} \circ r^{*}=r^{*} \circ f^{*}$, and by Proposition 2.8 we obtain the desired homotopy equivalence $g \circ r \simeq_{P} r \circ f$. By (b), the restriction of $r^{*}$ to the free group is an isomorphism, and thus $r$ is a homotopy equivalence of graphs.

The following proposition characterises the notion of reducibility.

Proposition 3.3. Let $[G, P, f]$ be a pattern with $|P|=n$. Then $[G, P, f]$ is reducible if and only if there exists $m<n$ with $n=q m$, for some $q \in \mathbb{Z}^{+} \backslash\{1\}$, such that for any $x \in P$ there exists a path $\gamma$ from $x$ to $f^{m}(x)$ satisfying:

$$
\left[\gamma\left(f^{m} \circ \gamma\right) \ldots\left(f^{(q-1) m} \circ \gamma\right)\right]=e_{x}
$$

where $e_{x}$ denotes the homotopy class of the trivial loop based at $x$.

Proof. Suppose that $[G, P, f]$ is reducible. Let $\left[G^{\prime}, Q, g\right]$ be a reduction of $[G, P, f]$ with $|Q|=m$, and let $r: G \longrightarrow G^{\prime}$ be the corresponding homotopy equivalence (recall that $r(P)=Q$ and $\left.g \circ r \simeq_{P} r \circ f\right)$. Notice that for each $x \in P, r\left(f^{m}(x)\right)=$ $g^{m}(r(x))=r(x)$.

First, we claim that there exists a unique $[\gamma] \in \pi\left(G,\left\{x, f^{m}(x)\right\}\right)$ such that $r^{*}([\gamma])=e_{r(x)}$. To prove the existence of such a $[\gamma]$, let $c$ be a path from $x$ to $f^{m}(x)$, and assume that $r^{*}([c])=[\beta] \neq e_{r(x)}$ for some $\beta \in \wp\left(G^{\prime},\{r(x)\}\right)$. Since $r^{*}: \pi(G,\{x\}) \longrightarrow \pi\left(G^{\prime},\{r(x)\}\right)$ is an isomorphism, there exists $\alpha \in \wp(G,\{x\})$ such that $r^{*}([\alpha])=[\beta]^{-1}$. So $r^{*}([\alpha] \cdot[c])=[\beta]^{-1} \cdot[\beta]=e_{r(x)}$. Then the desired $\gamma$ is any path from $x$ to $f^{m}(x)$ in the class of $[\alpha c]$. The uniqueness of $[\gamma]$ follows from the fact that $r^{*}$ is an isomorphism. This proves the claim.

Observe that $\delta=\gamma\left(f^{m} \circ \gamma\right) \ldots\left(f^{(q-1) m} \circ \gamma\right)$ is a loop based at $x$. In addition, since $r^{*} \circ f^{*}=g^{*} \circ r^{*}$, we have $r^{*}\left(\left[f^{i m} \circ \gamma\right]\right)=\left(g^{*}\right)^{i m}\left(r^{*}[\gamma]\right)=e_{r(x)}$. Hence $r^{*}([\delta])=e_{r(x)}$, and therefore $[\delta]=e_{x}$.

Now we prove the sufficiency of the conditions. For $i=0,1, \ldots, n-1$, let $x_{i}$ denote the point $f^{i}(x)$, and let $c_{i}$ be a path from $x_{0}$ to $x_{i}$. For $i=0,1, \ldots,(q-$ 1) $m-1$, let $d_{i}$ be the path $f^{i} \circ \gamma$.

By Proposition 2.3, $\left\{\left[d_{0}\right],\left[d_{1}\right], \ldots,\left[d_{(q-1) m-1}\right],\left[c_{1}\right], \ldots,\left[c_{m-1}\right]\right\}$ is a (free) system of path generators of $\pi(G, P)$. If $\left\{\delta_{1}, \ldots, \delta_{l}\right\}$ is a free system of generators of $\pi\left(G,\left\{x_{0}\right\}\right)$ then we obtain the following free system of generators of $\pi(G, P)$ :

$$
\left\{\delta_{1}, \ldots, \delta_{l},\left[d_{0}\right],\left[d_{1}\right], \ldots,\left[d_{(q-1) m-1}\right],\left[c_{1}\right], \ldots,\left[c_{m-1}\right]\right\}
$$

Notice that:

$$
\left\{\begin{aligned}
f^{*}\left(\left[d_{i}\right]\right) & =\left[d_{i+1}\right], \\
f^{*}\left(\left[d_{(q-1) m-1}\right]\right) & =\left[d_{(q-2) m}\right]^{-1} \ldots\left[d_{m}\right]^{-1} \cdot\left[d_{0}\right]^{-1} \\
f^{*}\left(\delta_{i}\right) & =\left[c_{1}\right]^{-1} \cdot \nu_{i}\left(\delta_{1}, \ldots, \delta_{l}\right) \cdot\left[c_{1}\right] \quad \text { for } i=1 \ldots, l \\
f^{*}\left(\left[c_{i}\right]\right) & =\left[c_{1}\right]^{-1} \cdot \omega_{i}\left(\delta_{1}, \ldots, \delta_{l}\right) \cdot\left[c_{i+1}\right] \text { for } i=1, \ldots, m-2, \text { and } \\
f^{*}\left(\left[c_{m-1}\right]\right) & =\left[c_{1}\right]^{-1} \cdot \omega_{m-1}\left(\delta_{1}, \ldots, \delta_{l}\right) \cdot\left[d_{0}\right] .
\end{aligned}\right.
$$

for some words $\omega_{i}$ and $\nu_{j}$ in $\delta_{1}, \ldots, \delta_{l}$.

Given $\left(G_{l}, A_{m}\right)$, we shall denote its distinguished vertex by $v_{0}$, its vertices by $v_{0}, \ldots, v_{m-1}$, its hairs by $\iota_{1}, \ldots, \iota_{m-1}$, and its petals by $\alpha_{1}, \ldots, \alpha_{l}$. We consider 
the $\operatorname{map} \varphi: \pi\left(G_{l}, A_{m}\right) \longrightarrow \pi\left(G_{l}, A_{m}\right)$ defined as follows:

$$
\begin{aligned}
\varphi\left(\left[\alpha_{i}\right]\right) & =\left[\iota_{1}\right]^{-1} \cdot \nu_{i}\left(\left[\alpha_{1}\right], \ldots,\left[\alpha_{l}\right]\right) \cdot\left[\iota_{1}\right] & & \text { for } i=i, \ldots, l, \\
\varphi\left(\left[\iota_{i}\right]\right) & =\left[\iota_{1}\right]^{-1} \cdot \omega_{i}\left(\left[\alpha_{1}\right], \ldots,\left[\alpha_{l}\right]\right) \cdot\left[\iota_{i+1}\right] & & \text { for } i=1, \ldots, m-2, \text { and } \\
\varphi\left(\left[\iota_{m-1}\right]\right) & =\left[\iota_{1}\right]^{-1} \cdot \omega_{m-1}\left(\left[\alpha_{1}\right], \ldots,\left[\alpha_{l}\right]\right) . & &
\end{aligned}
$$

Let $g:\left(G_{l}, A_{m}\right) \longrightarrow\left(G_{l}, A_{m}\right)$ be a topological realisation of $\varphi$, that is $g^{*}=\varphi$. Lastly, define $\phi: \pi(G, P) \longrightarrow \pi\left(G_{l}, A_{m}\right)$ by:

$$
\begin{aligned}
\phi\left(\delta_{i}\right) & =\left[\alpha_{i}\right] & & \text { for } i=i, \ldots, l, \\
\phi\left(\left[c_{i}\right]\right) & =\left[\iota_{i}\right] & & \text { for } i=1, \ldots, m-1, \text { and } \\
\phi\left(\left[d_{i}\right]\right) & =e_{g^{i}\left(x_{0}\right)} & & \text { for } i=1, \ldots,(q-1) m-1,
\end{aligned}
$$

where $e_{g^{i}\left(x_{0}\right)}$ denotes the homotopy class of the trivial loop based at $g^{i}\left(x_{0}\right)$.

By construction, $g^{*} \circ \phi=\phi \circ f^{*}$, and the restriction of $\phi$ to the free group $\pi\left(G_{l},\left\{x_{0}\right\}\right)$ is an isomorphism. It follows from Proposition 3.2 that $\left[G_{l}, A_{m}, g\right]$ is a reduction of $[G, P, f]$, and this completes the proof.

3.1. Patterns and Nielsen paths. The notion of Nielsen equivalence of fixed points will play a central rôle in the following section. Let $G$ be a graph, and let $x$ and $y$ be fixed points of a map $f \in \mathcal{C}(G, G)$. We say that $x$ and $y$ are Nielsen equivalent (written $x \sim_{f} y$ ) if there exists a path $\gamma$ from $x$ to $y$ such that $f \circ \gamma$ is homotopic to $\gamma$ keeping endpoints fixed. Such a path is called a Nielsen path. This defines an equivalence relation on the set of fixed points of $f$, and the corresponding equivalence classes are called fixed point classes. The following result shows that two Nielsen-equivalent fixed points of $f^{n}$ have essentially the same pattern.

Proposition 3.4. Let $f \in \mathcal{C}(G, G)$. Let $P$ and $Q$ be the orbits of two periodic points $x$ and $y$ of $f$. If $y$ is a fixed point of $f^{|P|}$ and $x \sim_{f|P|} y$, then $[G, Q, f] \preceq[G, P, f]$.

Proof. Let $\gamma$ be a Nielsen path from $x$ to $y$, and set $f^{i} \circ \gamma=\gamma_{i}$.

We begin by constructing a morphism $\phi: \pi(G, P) \longrightarrow \pi(G, Q)$. For any path $c_{i, j}$ between $f^{i}(x)$ and $f^{j}(x)$, define $\phi\left(\left[c_{i, j}\right]\right)=\left[\gamma_{i}^{-1} c_{i, j} \gamma_{j}\right]$. This is well defined because $\left[\gamma_{r}\right]=\left[\gamma_{s}\right]$ if $r$ and $s$ are congruent modulo $n$. It follows that:

$$
\phi\left(\left[c_{i, j}\right] \cdot\left[c_{j, k}\right]\right)=\left[\gamma_{i}^{-1} c_{i, j} c_{j, k} \gamma_{k}\right]=\left[\gamma_{i}^{-1} c_{i, j} \gamma_{j} \gamma_{j}^{-1} c_{j, k} \gamma_{k}\right]=\phi\left(\left[c_{i, j}\right]\right) \cdot \phi\left(\left[c_{j, k}\right]\right),
$$

and hence $\phi$ is indeed a morphism.

On the other hand, since $\phi$ maps a free system of generators of $\pi(G,\{x\})$ onto a free system of generators of $\pi(G,\{y\})$, the restriction of $\phi$ to this free group is an isomorphism.

Finally, we have that:

$$
f^{*}\left(\phi\left(\left[c_{i, j}\right]\right)\right)=f^{*}\left(\left[\gamma_{i}^{-1} c_{i, j} \gamma_{j}\right]\right)=\left[\gamma_{i+1}^{-1} f\left(c_{i, j}\right) \gamma_{j+1}\right]=\phi\left(f^{*}\left(\left[c_{i, j}\right]\right)\right),
$$

and the result follows from Proposition 3.2.

The above proposition says, in particular, that if $|P|=|Q|$ and $x$ and $y$ are Nielsen equivalent for $f^{|P|}$ then $(G, Q, f)$ and $(G, P, f)$ have the same pattern. The converse does not hold: to see this, it suffices to consider two non-Nielsen equivalent fixed points of a circle map.

Proposition 3.5. Let $[G, P, f]$ be a reducible pattern with $|P|=n$. Suppose that one of its reductions has period $m$, with $n=q m$ for some integer $q>1$. Then for each $x \in P,\left\{x, f^{m}(x), \ldots, f^{(q-2) m}(x), f^{(q-1) m}(x)\right\}$ is contained in a fixed point class of $f^{n}$. 
Proof. It suffices to show that $x \sim_{f^{n}} f^{m}(x)$ for any $x \in P$. Consider the path $\gamma$ from $x$ to $f^{m}(x)$ given by Proposition 3.3. Then we have:

$$
\left[\gamma\left(f^{m} \circ \gamma\right) \ldots\left(f^{(q-1) m} \circ \gamma\right)\right]=e_{x}
$$

where $e_{x}$ denotes the homotopy class of the trivial loop based on $x$. It follows that:

$$
\left[\left(f^{m} \circ \gamma\right) \ldots\left(f^{q m} \circ \gamma\right)\right]=e_{f^{m}(x)} .
$$

Thus we obtain:

$$
\left[f^{n} \circ \gamma\right]=\left[f^{q m} \circ \gamma\right]=\left[\left(f^{m} \circ \gamma\right) \ldots\left(f^{(q-1) m} \circ \gamma\right)\right]^{-1}=\gamma
$$

Hence $\gamma$ is a Nielsen path for $f^{n}$ from $x$ to $f^{m}(x)$.

In what follows, we describe a simple procedure to obtain a reducible pattern from a given pattern.

For $q \geq 3$, a $q$-star is a tree having a unique vertex of valence greater than 2 and $q$ valence-one vertices. A 2-star will be any tree homeomorphic to a closed interval of the real line. Let $f: G \longrightarrow G$ be a map, and let $x \in G$ be a periodic point of period $n$. Given $q \in \mathbb{Z}^{+} \backslash\{1\}$, consider the graph $G^{\prime}=G \cup\left(\cup_{i=0}^{n-1} S_{i}\right)$, where each $S_{i}$ is a $q$-star such that $G \cap S_{i}=\left\{f^{i}(x)\right\}$ and $f^{i}(x)$ has maximal valence in $S_{i}$, for $0 \leq i<n$.

For $i=0,1, \ldots, n-1$ and $j=1, \ldots, q$, let $x_{i, j}$ be the valence-one vertices of $S_{i}$. Let $Q$ be the union of all points $x_{i, j}$, and let $g:\left(G^{\prime}, Q\right) \longrightarrow(G, P)$ satisfy the following properties:

(i) $\left.g\right|_{G}=f$.

(ii) $g$ maps $S_{i}$ homeomorphically onto $S_{(i+1)} \bmod n$.

(iii) $g\left(x_{i, j}\right)=x_{i+1, j}$ for $i=0, \ldots, n-2$, and $g\left(x_{n-1, j}\right)=x_{0, j+1} \bmod q$.

We call $\left(G^{\prime}, Q, g\right)$ an extension of $(G, P, f)$. Clearly $[G, P, f]$ is a reduction of $[G, Q, g]$. Conversely, the following proposition shows that a (reducible) pattern may be considered to be an extension of any representative of one of its reductions.

Proposition 3.6. Let $(G, P, f),\left(G^{\prime}, Q, g\right) \in \Sigma^{\circ}$ be such that $\left[G^{\prime}, Q, g\right] \prec[G, P, f]$. Then there exists an extension $\left(G^{\prime \prime}, R, h\right)$ of $\left(G^{\prime}, Q, g\right)$ which satisfies $\left[G^{\prime \prime}, R, h\right]=$ $[G, P, f]$.

Proof. Let $n=|P|$ and $m=|Q|$ with $n=q m$ for some $q \in \mathbb{Z}^{+} \backslash\{1\}$. Let $x \in P$, and for $i=0, \ldots, n-1$, set $x_{i}=f^{i}(x)$. Let $r:(G, P) \longrightarrow\left(G^{\prime}, Q\right)$ be a homotopy equivalence between $G$ and $G^{\prime}$ satisfying $g \circ r \sim_{P} r \circ f$. From the proof of Proposition 3.3, there exist $m<n$ and a path $\gamma$ from $x_{0}$ to $x_{m}$ such that $r^{*}([\gamma])=e_{r(x)}$, and

$$
\left[\gamma\left(f^{m} \circ \gamma\right) \ldots\left(f^{(q-1) m} \circ \gamma\right)\right]=e_{x}
$$

where $e_{x}$ and $e_{r(x)}$ denote the homotopy classes of the trivial loops based at $x$ and $r(x)$ respectively.

Now for $i=0, \ldots,(q-1) m-1$, we denote the path $f^{i} \circ \gamma$ by $d_{i}$, and a free system of generators of $\pi\left(G,\left\{x_{0}\right\}\right)$ by $\left\{\delta_{1}, \ldots, \delta_{l}\right\}$. For $i=1, \ldots, m-1$, we choose a path $c_{i}$ from $x$ to $x_{i}$. As in the proof of Proposition 3.3, we choose:

$$
\left\{\delta_{1}, \ldots, \delta_{l},\left[d_{0}\right],\left[d_{1}\right], \ldots,\left[d_{(q-1) m-1}\right],\left[c_{1}\right], \ldots,\left[c_{m-1}\right]\right\}
$$

to be a free system of generators of $\pi(G, P)$. The induced morphism $f^{*}$ is given by equation (1).

For $i=1, \ldots, l$, set $\beta_{i}=r^{*}\left(\delta_{i}\right)$, and for $i=1, \ldots, m-1$, set $t_{i}=r\left(c_{i}\right)$. Since $r$ is a homotopy equivalence between $G$ and $G^{\prime}$ which collapses each $d_{i}$ to a point, $\left\{\beta_{1}, \ldots, \beta_{l}\right\}$ is a basis of for $\pi\left(G^{\prime},\left\{r\left(x_{0}\right)\right\}\right)$ and the paths $t_{i}$ are non degenerate. 
Thus $\left\{\beta_{1}, \ldots, \beta_{l},\left[t_{1}\right], \ldots,\left[t_{m-1}\right]\right\}$ is a free system of generators of $\pi\left(G^{\prime}, Q\right)$. Furthermore, we have that $g^{*} \circ r^{*}=r^{*} \circ f^{*}$. It follows that $g^{*}$ is given by:

$$
\begin{aligned}
g^{*}\left(\beta_{i}\right) & =\left[t_{1}\right]^{-1} \cdot \nu_{i}\left(\beta_{1}, \ldots, \beta_{l}\right) \cdot\left[t_{1}\right] & & \text { for } i=1, \ldots, l, \\
g^{*}\left(\left[t_{i}\right]\right) & =\left[t_{1}\right]^{-1} \cdot \omega_{i}\left(\beta_{1}, \ldots, \beta_{l}\right) \cdot\left[t_{i+1}\right] & & \text { for } i=1, \ldots, m-2, \text { and } \\
g^{*}\left(\left[t_{m-1}\right]\right) & =\left[t_{1}\right]^{-1} \cdot \omega_{m-1}\left(\beta_{1}, \ldots, \beta_{l}\right) \cdot\left[d_{0}\right], & &
\end{aligned}
$$

where $\omega_{i}$ and $\nu_{j}$ are the words used in the expression of $f^{*}$.

For $i=0, \ldots, m-1$, denote the points of $Q^{\prime}$ by $y_{i}=r\left(f^{i}(x)\right)$. Let $G^{\prime \prime}$ be the graph obtained by attaching a $q$-star at each $y_{i}$ as before. For $i=0, \ldots, m-1$ and $j=1, \ldots, q$, denote the valence-one vertices of $S_{i}$ by $z_{i}^{j}$, and the oriented edge from $y_{i}$ to $z_{i}^{j}$ by $s_{i}^{j}$. Let $R$ denote the union of all points $z_{i}^{j}$, and let $\left(G^{\prime \prime}, R, h\right)$ be the corresponding extension of $\left(G^{\prime}, Q, g\right)$. Set:

$$
\begin{aligned}
\eta & =\left(s_{0}^{1}\right)^{-1} s_{0}^{2}, & & \\
\eta_{i} & =h^{i}(\eta) & & \text { for } i=0, \ldots,(q-1) m-1, \\
b_{i} & =\left(s_{0}^{1}\right)^{-1} t_{i} s_{i}^{1} & & \text { for } i=1, \ldots, m-1, \text { and } \\
\alpha_{i} & =\left[s_{0}^{1}\right]^{-1} \cdot \beta_{i} \cdot\left[s_{0}^{1}\right] & & \text { for } i=1, \ldots, l .
\end{aligned}
$$

A direct computation shows that the corresponding expressions for $f^{*}$ and $h^{*}$ coincide, and we obtain the desired result.

\section{Persistence of patterns}

The aim of this section is to prove Theorem A which shows that the patterns of essential periodic orbits are preserved. Before doing this, we shall introduce some notation.

Let $G$ be a graph, and let $f \in \mathcal{C}(G, G)$. In what follows, if $x$ is a fixed point of $f$ then $[x, f]$ will denote the fixed point class of $x$ for $f$, and ind $[x ; f]$ will denote the index of $[x, f]$ with respect to $f$. That is, $\operatorname{ind}[x ; f]:=\operatorname{ind}([x, f], f)$ (see $[13])$.

Let $P$ be a periodic orbit of $f \in \mathcal{C}(G, G)$, and let $n$ be a multiple of $|P|$. We define the index of $P$ with respect to $f^{n}$, denoted by ind $\left[P ; f^{n}\right]$, to be the integer $\operatorname{ind}\left[x ; f^{n}\right]$ for each $x \in P$. The following lemma guarantees that this index is well defined.

Lemma 4.1. Let $P$ be a periodic orbit of $f \in \mathcal{C}(G, G)$. Let $n$ be a multiple of $|P|$, and let $x, y \in P$. Then ind $\left[x ; f^{n}\right]=\operatorname{ind}\left[y ; f^{n}\right]$.

Proof. Clearly there exists $j<|P|$ such that $y=f^{j}(x)$. In view of [13, Theorem I.5.2], we have that:

$$
\begin{aligned}
\operatorname{ind}\left[x ; f^{n}\right] & =\operatorname{ind}\left(\left[x, f^{n}\right], f^{n}\right)=\operatorname{ind}\left(\left[x, f^{n}\right], f^{n-j} \circ f^{j}\right) \\
& =\operatorname{ind}\left(f^{j}\left(\left[x, f^{n}\right]\right), f^{j} \circ f^{n-j}\right)=\operatorname{ind}\left(f^{j}\left(\left[x, f^{n}\right]\right), f^{n}\right),
\end{aligned}
$$

and $f^{j}\left(\left[x, f^{n}\right]\right)$ is a fixed point class of $f^{n}$. The lemma follows since $f^{j}\left(\left[x, f^{n}\right]\right)=$ $\left[y, f^{n}\right]$.

We recall that the periodic orbit $P$ is called essential if ind $\left[P ; f^{|P|}\right] \neq 0$.

The following results will play a crucial rôle in the proof of Theorem A.

Lemma 4.2. Let $f, g \in \mathcal{C}(G, G)$, and suppose that there exists a homotopy $\left\{h_{t}\right\}_{t=0}^{1}$ : $f \simeq g$. Then $h_{t}$ induces an index-preserving bijection $\kappa$ that, for each $n \in \mathbb{N}$, sends essential fixed point classes of $f^{n}$ to essential fixed point classes of $g^{n}$. Moreover, if $C$ is a fixed point class of $f^{n}, x \in C$ and $y \in \kappa(C)$ then there exists a path $\gamma(t)$ from $x$ to $y$ such that $h_{t}^{n}(\gamma(t)) \simeq \gamma(t)$, keeping endpoints fixed. 
The proof of this lemma is immediate from Theorems I.2.4 and I.4.5 of [13]. The following result asserts the preservation of the ordering $\preceq$ under homotopy.

Proposition 4.3. Let $(\Gamma, R, \varphi),(G, P, f) \in \Sigma^{\circ}$ be such that $[G, P, f] \preceq[\Gamma, R, \varphi]$ (so $|P|$ is a divisor of $n=|R|)$ and $\operatorname{ind}\left[P ; f^{n}\right] \neq 0$. Let $g \in \mathcal{C}(G, G)$ be homotopic to $f$. Then $\left[G, \operatorname{Orb}_{g}(y), g\right] \preceq[\Gamma, R, \varphi]$ for all $y \in \kappa\left(\left[x, f^{n}\right]\right)$ and $x \in P$, where $\kappa$ is the bijection given by Lemma 4.2 for $f$ and $g$.

Observe that in this proposition the assumption is on the orbit $P$, but for the period of $R$. In particular we do not suppose that $P$ is an essential orbit.

Proof of Proposition 4.3. Since $[G, P, f] \preceq[\Gamma, R, \varphi]$, there exists a homotopy equivalence $r: \Gamma \longrightarrow G$ such that $r \circ \varphi \simeq_{R} f \circ r$ (in particular, this implies that $\left.r \circ \varphi\right|_{R}=$ $\left.\left.f \circ r\right|_{R}\right)$. Let $\left\{h_{t}\right\}_{t=0}^{1}$ be a homotopy between $f$ and $g$. Clearly, $\left\{h_{t}^{n}\right\}_{t=0}^{1}$ is a homotopy from $f^{n}$ to $g^{n}$.

Pick a point $z$ of $R$. Since $\operatorname{ind}\left[r(z) ; f^{n}\right]=\operatorname{ind}\left[P ; f^{n}\right] \neq 0$, by Lemma 4.2 we have that $\kappa\left(\left[r(z), f^{n}\right]\right)$ is a fixed point class of $g^{n}$ for which:

$$
\operatorname{ind}\left(\kappa\left(\left[r(z), f^{n}\right]\right), g^{n}\right)=\operatorname{ind}\left[P ; f^{n}\right] \neq 0 .
$$

Take $y \in \kappa\left(\left[r(z), f^{n}\right]\right)$, and set $Q=\operatorname{Orb}_{g}(y)$. Then $|Q|$ is a divisor of $n$, and $\operatorname{ind}\left[Q ; g^{n}\right] \neq 0$.

We have to prove that $[G, Q, g] \preceq[\Gamma, R, \varphi]$. In view of Proposition 3.2, we need to find a groupoid morphism

$$
\phi: \pi(\Gamma, R) \longrightarrow \pi(G, Q)
$$

such that $\phi: \pi(\Gamma,\{z\}) \longrightarrow \pi(G,\{y\})$ is a free group isomorphism and $g^{*} \circ \phi=\phi \circ \varphi^{*}$. Let us construct such a morphism $\phi$. By Lemma 4.2, there exists a path $\sigma$ in $G$ from $r(z)$ to $y$ such that the path $h_{t}^{n}(\sigma(t))$ is homotopic to $\sigma$ with endpoints fixed. Let us write $R=\left\{z_{i}=\varphi^{i}(z): i=0,1, \ldots, n-1\right\}, P=\left\{x_{i}=f^{i}(r(z))=r\left(z_{i}\right): i=\right.$ $0,1, \ldots, n-1\}$, and $Q=\left\{y_{i}=g^{i}(y): i=0,1, \ldots, n-1\right\}$ (notice that $|Q|$ and $|P|$ may be less than $n)$. For $i=0,1, \ldots, n-1$ and $t \in[0,1]$, we define $\sigma_{i}(t)=h_{t}^{i}(\sigma(t))$. So $\sigma_{i}$ is a path from $x_{i}$ to $y_{i}$, and $\sigma_{i+1}(t)=h_{t}\left(\sigma_{i}(t)\right)$ for $i=0,1, \ldots, n-2$ and $t \in[0,1]$. The fact that $h_{t}^{n}(\sigma(t))$ is homotopic to $\sigma$ implies that $h_{t}\left(\sigma_{n-1}(t)\right)$ is homotopic to $\sigma(t)=\sigma_{0}(t)$ with endpoints fixed.

Let $\left[c_{i, j}\right] \in \pi(\Gamma, R)$ be a class of paths from $z_{i}$ to $z_{j}$ for some $i, j \in\{0,1, \ldots, n-1\}$. We define the $\operatorname{map} \phi: \pi(\Gamma, R) \longrightarrow \pi(G, Q)$ by:

$$
\phi\left(\left[c_{i j}\right]\right)=\left[\sigma_{i}^{-1}\left(r \circ c_{i j}\right) \sigma_{j}\right] .
$$

It is a well-defined groupoid morphism (even when $|Q|<n$ ). Moreover, $\left.\phi\right|_{\pi(\Gamma,\{z\})}$ is given by:

$$
\phi([c])=\left[\sigma^{-1}(r \circ c) \sigma\right]=[\sigma]^{-1} r^{*}([c])[\sigma],
$$

where $c$ is any loop based at $z$ in $\Gamma$. Since $r$ is a homotopy equivalence, $r^{*}$ and hence $\left.\phi\right|_{\pi(\Gamma,\{z\})}$ are free group isomorphisms.

To complete the proof of the proposition, we have to check that $g^{*} \circ \phi=\phi \circ \varphi^{*}$. That is, for each $z_{i}, z_{j}$ and each path $c_{i j}$ from $z_{i}$ to $z_{j}$, we have to show that the path $g\left(\sigma_{i}^{-1}\left(r \circ c_{i j}\right) \sigma_{j}\right)$ is homotopic to $\sigma_{i+1}^{-1}(\bmod n)\left(r \circ \varphi \circ c_{i j}\right) \sigma_{j+1}(\bmod n)$ with endpoints fixed (see Lemma 2.5(a)). Since $r \circ \varphi \circ c_{i j} \simeq f \circ r \circ c_{i j}$ with endpoints fixed, it is enough to show that

$$
\alpha:=g\left(\sigma_{i}^{-1}\left(r \circ c_{i j}\right) \sigma_{j}\right)=g\left(\sigma_{i}^{-1}\right) g\left(r \circ c_{i j}\right) g\left(\sigma_{j}\right)
$$

is homotopic to

$$
\beta:=\sigma_{i+1(\bmod n)}^{-1} f\left(r \circ c_{i j}\right) \sigma_{j+1(\bmod n)}
$$

with endpoints fixed.

Let us construct a homotopy between $\alpha$ and $\beta$ in two parts (see Figure 2). 


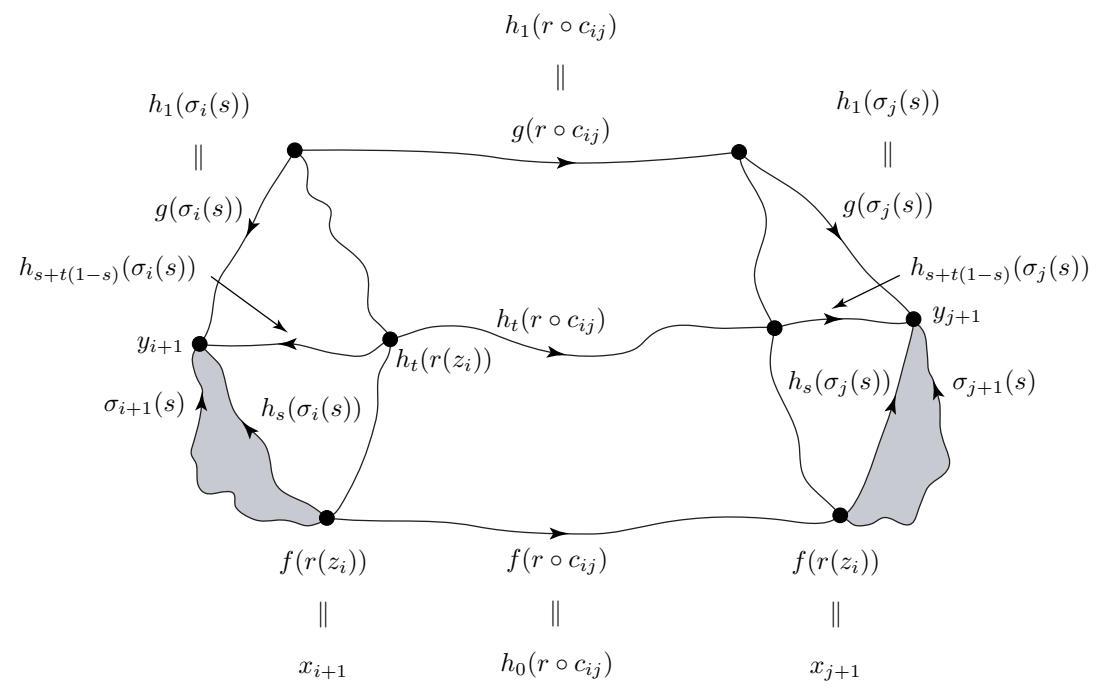

Figure 2. The situation from the proof of Proposition 4.3.

Part 1. $h_{t}\left(r \circ c_{i j}\right)$ is a homotopy between $f\left(r \circ c_{i j}\right)$ and $g\left(r \circ c_{i j}\right)$. Given $t \in[0,1]$, note that the endpoints of $h_{t}\left(r \circ c_{i j}\right)$ are $h_{t}\left(r\left(z_{i}\right)\right)=h_{t}\left(x_{i}\right)$ and $h_{t}\left(r\left(z_{j}\right)\right)=h_{t}\left(x_{j}\right)$.

Part 2. For $i \in\{0,1, \ldots, n-1\}$, we consider the homotopy $h_{s+t(1-s)}\left(\sigma_{i}(s)\right), \quad t, s \in$ $[0,1]$, which for $t=0$ gives $h_{s}\left(\sigma_{i}(s)\right)=\sigma_{i+1}(s)$, and for $t=1$, gives $h_{1}\left(\sigma_{i}(s)\right)=$ $g\left(\sigma_{i}(s)\right)$. If $t \in[0,1]$, the endpoints of $h_{s+t(1-s)}\left(\sigma_{i}(s)\right)$ are $h_{t}\left(\sigma_{i}(0)\right)=h_{t}\left(x_{i}\right)$ and $h_{1}\left(\sigma_{i}(1)\right)=h_{1}\left(y_{i}\right)=g\left(y_{i}\right)=y_{i+1}$. In particular this homotopy fixes the right endpoint.

Using these two homotopies, we see that $\alpha$ is homotopic to $h_{s}\left(\sigma_{i}(s)\right)^{-1} f(r \circ$ $\left.c_{i j}\right) h_{s}\left(\sigma_{j}(s)\right)$ with endpoints fixed. If $i, j \neq n-1$, the latter is equal to $\beta$, and we obtain the desired homotopy. Finally, if either $i$ or $j$ is equal to $n-1$ then $h_{s}\left(\sigma_{i}(s)\right)^{-1} f\left(r \circ c_{i j}\right) h_{s}\left(\sigma_{j}(s)\right)$ is homotopic to $\beta$ because, by the definition of $\sigma$, $h_{s}\left(\sigma_{n-1}(s)\right)$ is homotopic to $\sigma_{0}(s)$ with endpoints fixed.

Lemma 4.4. Let $r: G \longrightarrow G^{\prime}$ be a homotopy equivalence, let $\varphi \in \mathcal{C}\left(G^{\prime}, G\right)$, and let $P$ be a periodic orbit of $\varphi \circ r$. Then $r(P)$ is a periodic orbit of $r \circ \varphi$, and $[G, \varphi \circ r, P]=\left[G^{\prime}, r \circ \varphi, r(P)\right]$. Moreover, $\operatorname{ind}\left[P ;(\varphi \circ r)^{|P|}\right]=\operatorname{ind}\left[r(P),(r \circ \varphi)^{|P|}\right]$.

Proof. Set $n=|P|$ and $\widetilde{\varphi}=(\varphi \circ r)^{n-1} \circ \varphi$. Observe that $r \circ \widetilde{\varphi}=(r \circ \varphi)^{n}$ and $\widetilde{\varphi} \circ r=(\varphi \circ r)^{n}$. Thus $P$ is a set of fixed points of $\widetilde{\varphi} \circ r$, and consequently $r(P)$ is a set of fixed points of $r \circ \widetilde{\varphi},\left.r\right|_{P}$ is injective and $r \circ \varphi(r(P))=r(P)$. Therefore $[G, \varphi \circ r, P]=\left[G^{\prime}, r \circ \varphi, r(P)\right]$, since the following diagram:

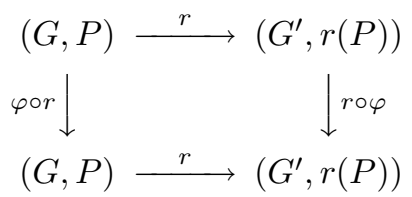

commutes (exactly). Further, from [13, Theorem I.5.2] we obtain:

$$
\operatorname{ind}\left[P ;(\varphi \circ r)^{n}\right]=\operatorname{ind}[x ; \widetilde{\varphi} \circ r]=\operatorname{ind}[r(x) ; r \circ \widetilde{\varphi}]=\operatorname{ind}\left[r(P),(r \circ \varphi)^{n}\right],
$$

where $x$ is a point of $P$.

Proof of Theorem A. Let $f: G \longrightarrow G$ and $g: G^{\prime} \longrightarrow G^{\prime}$ be two representatives of the same endomorphism. Then there exists a homotopy equivalence $r: G \longrightarrow G^{\prime}$ and $s: G^{\prime} \longrightarrow G$ satisfying $r \circ s \simeq \operatorname{Id}_{G^{\prime}}, s \circ r \simeq \operatorname{Id}_{G}$ and $r \circ f \simeq g \circ r$. Let 
$\widehat{f}=s \circ g \circ r \in \mathcal{C}(G, G)$, and let $\widehat{g}=r \circ s \circ g \in \mathcal{C}\left(G^{\prime}, G^{\prime}\right)$. Then $\widehat{f} \simeq s \circ r \circ f \simeq f$ and $\widehat{g} \simeq g$.

Now let $P$ be an essential periodic orbit of period $n$ of $f$, so ind $\left[P ; f^{n}\right] \neq 0$. Set $\psi=\widehat{f}^{n-1} \circ s \circ g$. Then $\psi$ satisfies $\widehat{f}^{n}=\psi \circ r$ and:

$$
r \circ \psi=r \circ(s \circ g \circ r)^{n-1} \circ s \circ g=\widehat{g}^{n} .
$$

We now consider the following diagram which commutes up to homotopy:

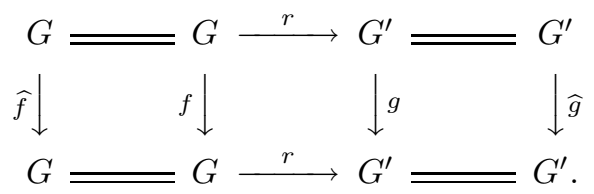

The strategy of the proof is as follows:

(a) we compare the given essential periodic orbit $P$ of $f$ with the corresponding orbit $\widehat{P}$ of $\widehat{f}$ given by a weak version of Proposition 4.3 .

(b) Then we compare $\widehat{P}$ with the corresponding orbit $\widehat{Q}$ of $\widehat{g}$ given by the commutativity property of Lemma 4.4 .

(c) Finally we apply Proposition 4.3 once more to $\widehat{Q}$ and the corresponding orbit $Q$ of $g$.

We now give the details of these three steps. For step (a), we apply Proposition 4.3 with $(\Gamma, R, \varphi)=(G, P, f)$. Then $[\Gamma, R, \varphi]=[G, P, f]$ and ind $\left[P ; f^{n}\right] \neq 0$. The proposition implies that there is an index-preserving bijection $\kappa_{a}$ between the essential fixed point classes of $f^{n}$ and those of $\widehat{f}^{n}$. Let $x \in P, y \in \kappa_{a}\left(\left[x, f^{n}\right]\right)$ and $\widehat{P}=\operatorname{Orb}_{\widehat{f}}(y)$. Then $[G, \widehat{P}, \widehat{f}] \preceq[G, P, f]$.

For step (b), first notice that the maps $\psi$ and $r$ satisfy the assumptions of Lemma 4.4. Therefore $\left[r(y), \widehat{g}^{n}\right]$ is an essential fixed point class of $\widehat{g}^{n}$. Applying this lemma, we see that $\left[G^{\prime}, \widehat{Q}, \widehat{g}\right]=[G, \widehat{P}, \widehat{f}]$, where $\widehat{Q}=\operatorname{Orb}_{\widehat{g}}(r(y))$, and ind $\left[\widehat{Q} ; \widehat{g}^{n}\right] \neq 0$. It follows from above that $\left[G^{\prime}, \widehat{Q}, \widehat{g}\right] \preceq[G, P, f]$.

Finally, for step (c), applying Proposition 4.3 to $g$ and $\widehat{g}$, we see that there exists a bijection $\kappa_{c}$ between the essential fixed point classes of $\widehat{g}^{n}$ and those of $g^{n}$. Now set $Q=\operatorname{Orb}_{g}(w)$, where $w \in \kappa_{c}\left(\left[r(y), \widehat{g}^{n}\right]\right)$. Proposition 4.3 implies that $\left[G^{\prime}, Q, g\right] \preceq[G, P, f]$. This completes the proof of the theorem.

The following examples show that Theorem A is in some sense the best possible. In Example 4.5 we construct a reducible pattern having a representative with an essential periodic orbit, and an equivalent model which exhibits one of its reductions. In Example 4.6 we show that there exist inessential periodic orbits that are not realised in an equivalent model. Although the first example is given in the simple framework of interval maps, the important part of the phenomenon is already exhibited. Other examples may be easily be constructed in the setting of honest graphs (with loops), but they will necessarily be more complicated without displaying any essentially new feature.

Example 4.5. Let $I$ be a closed interval of the real line. Let $f \in \mathcal{C}(I, I)$ be a map having a periodic orbit $P$ of period $n \geq 2$. Take $x \in P$, and denote its fixed point class with respect to $f^{n}$ by $F$. Then $F$ contains all fixed points of $f^{n}$, and its index is equal to -1 . Further, $[I, P, f]$ is reducible. Let $g \in \mathcal{C}(I, I)$ be such that $g(x) \leq x$ for all $x \in I$. This map has no periodic orbits other than fixed points, and is homotopic to $f$. Hence the class $F$ is associated to the class of fixed points of $g$. Then the pattern $[I, P, f]$ does not belong to the set of patterns of the map $g$. 
Example 4.6. Let $f$ be a degree one circle map with periodic orbits, and let $g$ be an irrational rotation of the circle. Since $f$ and $g$ are homotopic this implies that all fixed point classes of $f^{n}$ have index 0 .

\section{EFFICIENT MODELS}

A graph map $f: G \longrightarrow G$ will be said to possess an invariant forest if there exists an invariant subgraph whose connected components are trees.

Recall that a topological representative $f: G \longrightarrow G$ for $\Phi$ is said to be efficient if it has no invariant forests, $G$ has no valence-one vertices, and if for all $k \in \mathbb{N}$, the restriction of $f^{k}$ to the interior of each edge of $G$ is locally injective.

A precise definition of index is given in [13], although the reader should bear in mind that the index considered in this paper is minus that defined by Jiang. The index of a fixed point $x$ of a map $f$ will be denoted by ind $(x, f)$.

If $f$ is an efficient, expanding map then each fixed point of $f^{n}$ with $n \in \mathbb{N}$ is an isolated fixed point. Hence each fixed point class of $f^{n}$ is finite, and the index of the class is just the sum of the indices for each fixed point in the class. The notion of index in our context of graph maps has the following geometric interpretation. Let $x$ be fixed under $f^{n}$, and let $U_{x}$ be an open neighbourhood of $x$ in $G$ whose closure is homeomorphic to a tree (a $d(x)$-star). Let $E$ be the set of edges $e$ of $U_{x}$ that contain an interval $I$ with endpoint $x$ and such that $f^{n}(I)=e$. Then $\operatorname{ind}\left(x, f^{n}\right)$ satisfies:

$$
-1 \leq \operatorname{ind}\left(x, f^{n}\right)=\operatorname{Card}(E)-1 \leq d(x)-1 .
$$

The following lemma allows us to estimate the index of fixed point classes in efficient, expanding models.

Lemma 5.1. Let $f \in \mathcal{C}(G, G)$ be an efficient, expanding map, and let $F$ be a fixed point class of $f^{n}$. If $F$ has just one point which is not a vertex then $\operatorname{ind}\left(F, f^{n}\right)= \pm 1$. If the cardinal of $F$ is greater than one then ind $\left(x, f^{n}\right)=1$ for all $x \in F \backslash V(G)$, $\operatorname{ind}\left(x, f^{n}\right) \geq 0$ for all $x \in F \cap V(G)$, and

$$
\operatorname{ind}\left(F, f^{n}\right) \geq \operatorname{Card}(F)-\operatorname{Card}(F \cap V(G)) .
$$

Proof. If $F=\{x\}$, since $f$ is efficient and expanding, the first statement of the lemma follows from (2). So suppose that the cardinal of $F$ is greater than one, and let $x, y \in F, y \neq x$. Let $\rho$ be a Nielsen path of $f^{n}$ from $x$ to $y$. Without loss of generality, we may assume that this Nielsen path is indivisible, in other words, there is no Nielsen path $\gamma$ for $f^{n}$ contained in $\rho$. From [7, Lemma 3.4] there are paths $\alpha, \beta$ and $\tau$ such that $\rho=\alpha \beta, f^{n} \circ \alpha=\alpha \tau$ and $f^{n} \circ \beta=\tau^{-1} \beta$. Note that in [7] it is implicitly assumed that $f$ is induced by a free group automorphism. However to obtain the above properties of Nielsen paths this assumption is not used. Since $f^{n}$ linearly expands each edge, it follows that ind $\left(x, f^{n}\right) \geq 0$ if $x \in F \cap V(G)$, and $\operatorname{ind}\left(x, f^{n}\right)=1$ otherwise. Thus $\sum_{x \in F \cap V(G)} \operatorname{ind}\left(x, f^{n}\right) \geq 0$.

Since $f$ is efficient and expanding, $\operatorname{Card}(F)$ is finite, and hence:

$$
\operatorname{ind}\left(F, f^{n}\right)=\sum_{x \in F} \operatorname{ind}\left(x, f^{n}\right)=\sum_{x \in F \backslash V(G)} \operatorname{ind}\left(x, f^{n}\right)+\sum_{x \in F \cap V(G)} \operatorname{ind}\left(x, f^{n}\right) .
$$

From above,

$$
\sum_{x \in F \backslash V(G)} \operatorname{ind}\left(x, f^{n}\right)=\operatorname{Card}(F)-\operatorname{Card}(F \cap V(G)),
$$

and the result follows. 
Given an efficient, expanding map $f \in \mathcal{C}(G, G)$, let $\mathcal{D}_{f}$ be the set of all points $x \in G$ satisfying the following property: there exist $y \in G, y \neq x$, and a nonnegative integer $n$ such that $x$ and $y$ are Nielsen-equivalent fixed points of $f^{n}$. The number of Nielsen paths for all the iterates of $f$ is directly related to $\operatorname{Card}\left(\mathcal{D}_{f}\right)$, and as we show in following proposition, it is finite and bounded above by $\operatorname{Card}(V(G))$ and the Euler characteristic $\chi(G)$ of $G$.

Proposition 5.2. Let $f \in \mathcal{C}(G, G)$ be an efficient, expanding map. Then the set $\mathcal{D}_{f}$ is finite. Moreover,

$$
\operatorname{Card}\left(\mathcal{D}_{f}\right) \leq 2(\operatorname{Card}(V(G))-2 \chi(G))
$$

Proof. From [14, Theorem 1] we see that:

$$
\sum_{\operatorname{ind}\left(F, f^{n}\right)>1}\left(\operatorname{ind}\left(F, f^{n}\right)-1\right) \leq-2 \chi(G) .
$$

for all $n \in \mathbb{N}$. From Lemma 5.1, a periodic point $x \notin \mathrm{V}(G)$ either belongs to $\mathcal{D}_{f}$, or it is alone in its Nielsen class, in which case its index is less than or equal to 1 , and thus it does not appear in the above inequality. Thus for a Nielsen class to appear in the above inequality, either it contains at least two points of $\mathcal{D}_{f}$, or must contains an element of $\mathrm{V}(G)$. By the above inequality the number of such classes is bounded. Further, their cardinality is also bounded by Lemma 5.1. Thus, $\operatorname{Card}\left(\mathcal{D}_{f}\right)$ is finite. An easy computation shows that:

$$
\operatorname{Card}\left(\mathcal{D}_{f}\right) \leq 2(\operatorname{Card}(V(G))-2 \chi(G))
$$

Given $f \in \mathcal{C}(G, G)$, the following theorem asserts the finiteness of the set of all periodic orbits of $f$ such that either $(G, P, f)$ is inessential or $[G, P, f]$ is reducible.

Theorem 5.3. Let $f \in \mathcal{C}(G, G)$ be an efficient, expanding map. Then for almost all periodic points $x$ of $f,\left(G, \operatorname{Orb}_{f}(x), f\right)$ is essential and $\left[G, \mathrm{Orb}_{f}(x), f\right]$ is irreducible, the number of exceptional points being at most $3 \operatorname{Card}(V(G))-4 \chi(G)$.

Proof. If $\left[G, \operatorname{Orb}_{f}(x), f\right]$ is reducible then $x \in \mathcal{D}_{f}$ by Proposition 3.5. Further, if $\operatorname{Orb}_{f}(x)$ is inessential then $x \in V(G)$ from Lemma 5.1. The result follows from Proposition 5.2.

The exceptional points in the above theorem correspond either to inessential periodic orbits of vertices or to reducible periodic orbits. The following example shows that both situations can occur for efficient, expanding maps that are topological representatives of irreducible endomorphisms.

Example 5.4. Let $G$ be the graph shown in Figure 3, and let $f: G \longrightarrow G$ be defined by:

$$
\begin{aligned}
f\left(a_{1}\right) & =a_{2}, \\
f\left(a_{2}\right) & =a_{6} a_{3}, \\
f\left(a_{3}\right) & =a_{5} a_{1}, \\
f\left(a_{4}\right) & =a_{1} a_{2} a_{6} a_{3} a_{1}, \\
f\left(a_{5}\right) & =a_{4} a_{3} a_{1}, \\
f\left(a_{6}\right) & =a_{1} .
\end{aligned}
$$

Since $f$ is a positive endomorphism, for all $n>0$, there are no cancellations in the algebraic expression of $f^{n}$, and thus $f^{n}$ restricted to any edge is locally injective. Since there are no invariant forests, $f$ is efficient. 
$a_{4}$

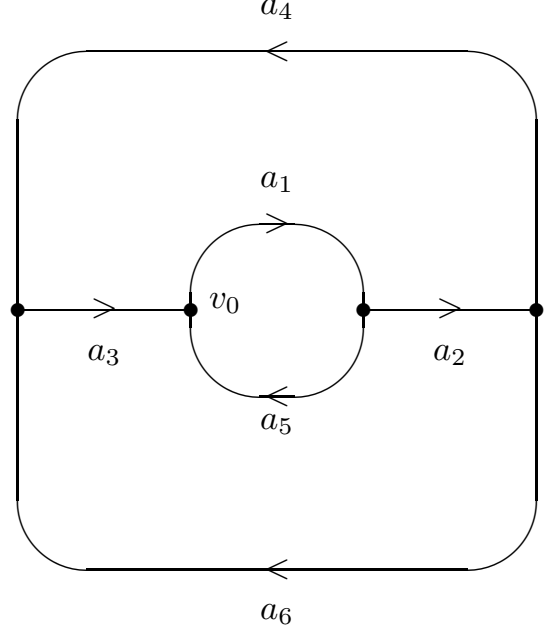

Figure 3. The graph $G$ of Example 5.4

Consider the following generators of $\pi\left(G,\left\{v_{0}\right\}\right)$ :

$$
\begin{aligned}
& \alpha_{1}=a_{1} a_{2} a_{6} a_{3} a_{1} a_{5}, \\
& \alpha_{2}=a_{1} a_{2} a_{4} a_{6}^{-1} a_{2}^{-1} a_{1}^{-1}, \\
& \alpha_{3}=a_{1} a_{2} a_{6} a_{3},
\end{aligned}
$$

and choose $a_{1}$ to be a path from $v_{0}$ to its image. With this choice, the induced endomorphism $f^{*}: \pi\left(G, v_{0}\right) \longrightarrow \pi\left(G, v_{0}\right)$ is given by:

$$
\begin{aligned}
& f^{*}\left(\left[\alpha_{1}\right]\right)=\left[\alpha_{1}\right]\left[\alpha_{2}\right]\left[\alpha_{3}\right], \\
& f^{*}\left(\left[\alpha_{2}\right]\right)=\left[\alpha_{3}\right], \\
& f^{*}\left(\left[\alpha_{3}\right]\right)=\left[\alpha_{1}\right] .
\end{aligned}
$$

Clearly $f^{*}$ is an irreducible automorphism of $\mathbb{F}_{3}$. Thus $f$ is an efficient representative of an irreducible automorphism of $\mathbb{F}_{3}$.

On the other hand, there exists a periodic orbit $P$ of $f$ of period 2 whose points, denoted respectively by $p$ and $q$, lie in $a_{3}$ and $a_{5}$. Let $\omega$ be the oriented injective subpath of $a_{3}$ from $p$ to $v_{0}$, and let $\pi$ be the oriented injective subpath of $\bar{a}_{5}$ from $v_{0}$ to $q$. Direct computations show that $f(\omega \pi)=\bar{\pi} a_{1} \bar{a}_{1} \bar{\omega}$, and thus $[G, P, f]$ is reducible from Proposition 3.3. The orbit $\{p, q\}$ is essential because $\operatorname{ind}\left(F, f^{2}\right)=2$, where the fixed point class of $p$ is denoted by $F$.

Another efficient representative of $f^{*}$ may be obtained by considering the map $g: G^{\prime} \longrightarrow G^{\prime}$, where $G^{\prime}$ is the rose with three petals $\alpha, \beta$ and $\gamma$, given by:

$$
\begin{aligned}
& g(\alpha)=\alpha \beta \gamma, \\
& g(\beta)=\gamma, \\
& g(\gamma)=\alpha,
\end{aligned}
$$

which is also efficient.

Notice that this representative has an inessential periodic orbit of vertices (in fact, a fixed point), while the preceding representative $f: G \longrightarrow G$ has no fixed points. So we have an example of vanishing inessential fixed points in efficient models.

Since the orbit $\{p, q\}$ of $f$ is essential, by Theorem A there exists a fixed point class $C$ of $g^{2}$ that is associated with the class $F$. Since $g$ has no periodic orbits of period 2, $C$ must be the class of the fixed point. We thus obtain an example of a reducible pattern in an efficient model that is reduced by a homotopy equivalence. 
We are now ready to state and prove the minimality (within the homotopy class) of the set of periodic orbits of its efficient representatives.

Theorem 5.5. Let $f \in \mathcal{C}(G, G)$ be an efficient, expanding map. Then there exists a cofinite subset $\mathcal{B}$ of the set of periodic orbits of $f$ with the property that, for each $\left(G^{\prime}, g\right)$ equivalent to $(G, f)$, there exists a pattern-preserving injective map from $\mathcal{B}$ to the set of periodic orbits of $g$. Moreover, the number of periodic points of $f$ whose orbit does not belong to $\mathcal{B}$ is at most $3 \operatorname{Card}(V(G))-4 \chi(G)$.

Proof. We define $\mathcal{B}=\left\{\operatorname{Orb}_{f}(x): x\right.$ is periodic and $\left.x \notin \mathcal{D}_{f} \cup \mathrm{V}(G)\right\}$. From Proposition 5.2 it follows that the number of periodic points of $f$ whose orbit does not belong to $\mathcal{B}$ is at most $3 \operatorname{Card}(V(G))-4 \chi(G)$. For each $P \in \mathcal{B}$, it follows from the proof of Theorem 5.3 that $P$ is essential and $[G, P, f]$ is irreducible.

We now define a map $\iota$ from $\mathcal{B}$ as follows. For each $P \in \mathcal{B}$, choose $x \in P$ and $z \in \kappa\left(\left[x, f^{|P|}\right]\right)$, where $\kappa$ is the map given by Theorem A. Then we define $\iota(P)=\operatorname{Orb}_{g}(z)$, and the result follows from Theorem A.

As a corollary of this theorem, we obtain Theorem B.

Proof of Theorem B. Since all vertices of an efficient representative have valence greater than 2, using the notation and the proof of Proposition 5.2, we obtain:

$$
\operatorname{Card}(\mathrm{V}(G))=\sum_{k=3}^{\Delta} \nu_{k} \leq \sum_{k=3}^{\Delta}(k-2) \nu_{k}=-2 \chi(G)=2(n-1) .
$$

Thus $3 \operatorname{Card}(V(G))-4 \chi(G) \leq 10(n-1)$. The result follows from Theorem 5.5.

\section{REFERENCES}

1. Ll. Alsedà, J. Guaschi, J. Los, F. Mañosas, and P. Mumbrú, Canonical representatives for patterns of tree maps, Topology 36 (1997), no. 5, 1123-1153. MR 99f:58062

2. Ll. Alsedà, J. Llibre, and M. Misiurewicz, Combinatorial dynamics and entropy in dimension one, second ed., World Scientific Publishing Co. Inc., River Edge, NJ, 2000. MR 2001j:37073

3. Ll. Alsedà, F. Mañosas, and P. Mumbrú, Minimizing topological entropy for continuous maps on graphs, Ergodic Theory Dynam. Systems 20 (2000), no. 6, 1559-1576. MR 2001k:37023

4. D. Asimov and J. Franks, Unremovable closed orbits, (1993), preprint. (This is a revised version of an article which appeared in Springer Lecture Notes in Mathematics 1007, SpringerVerlag, Berlin, 1983).

5. S. Baldwin, Generalizations of a theorem of Sarkovskii on orbits of continuous real-valued functions, Discrete Math. 67 (1987), no. 2, 111-127. MR 89c:58057

6. G. Besson, G. Courtois, and S. Gallot, Les variétés hyperboliques sont des minima locaux de l'entropie topologique, Invent. Math. 117 (1994), no. 3, 403-445. MR 95d:53034

7. M. Bestvina and M. Handel, Train tracks and automorphisms of free groups, Ann. of Math. (2) 135 (1992), no. 1, 1-51. MR 92m:20017

8. P. L. Boyland, Braid types and a topological method of proving positive entropy, (1984), preprint.

9. R. Brown, Topology, second ed., Ellis Horwood Ltd., Chichester, 1988, A geometric account of general topology, homotopy types and the fundamental groupoid. MR 90k:54001

10. W. Dicks and E. Ventura, The group fixed by a family of injective endomorphisms of a free group, American Mathematical Society, Providence, RI, 1996. MR 97h:20030

11. A. Fathi, F. Laudenbach, and V. Poénaru, Travaux de Thurston sur les surfaces, Astérisque 66-67 (1979).

12. T. Hall, Unremovable periodic orbits of homeomorphisms, Math. Proc. Cambridge Philos. Soc. 110 (1991), no. 3, 523-531. MR 92i:58146

13. B. Jiang, Lectures on Nielsen fixed point theory, American Mathematical Society, Providence, R.I., 1983. MR 84f:55002

14. MR 99h:55002 
15. J. Los, On the conjugacy problem for automorphisms of free groups, Topology 35 (1996), no. 3, 779-808, With an addendum by the author. MR 97h:20031

16. - On the forcing relation for surface homeomorphisms, Inst. Hautes Études Sci. Publ. Math. (1997), no. 85, 5-61. MR 98h:58151

17. M. Lustig, Structure and conjugacy for automorphisms of free groups i, ii, (2001), preprint.

18. T. Matsuoka, Braids of periodic points and a 2-dimensional analogue of Sharkovskiı's ordering, Dynamical systems and nonlinear oscillations (Kyoto, 1985), World Sci. Publishing, Singapore, 1986, pp. 58-72. MR 88b:58114

19. M. Misiurewicz and Z. Nitecki, Combinatorial patterns for maps of the interval, Mem. Amer. Math. Soc. 94 (1991), no. 456, vi+112. MR 92h:58105

20. G. D. Mostow, Quasi-conformal mappings in n-space and the rigidity of hyperbolic space forms, Inst. Hautes Études Sci. Publ. Math. (1968), no. 34, 53-104. MR 38 \#4679

21. Z. Sela, The isomorphism problem for hyperbolic groups. I, Ann. of Math. (2) 141 (1995), no. 2, 217-283. MR 96b:20049

22. A. N. Sharkovskiur, Coexistence of cycles of a continuous map of the line into itself, Proceedings of the Conference "Thirty Years after Sharkovskiı̌'s Theorem: New Perspectives" (Murcia, 1994), Internat. J. Bifur. Chaos Appl. Sci. Engrg. 5 (1995), no. 5, 1263-1273, Translated from the Russian [Ukrain. Mat. Zh. 16 (1964), no. 1, 61-71; MR 28 \#3121] by J. Tolosa. MR 96j:58058

23. W. P. Thurston, On the geometry and dynamics of diffeomorphisms of surfaces, Bull. Amer. Math. Soc. (N.S.) 19 (1988), no. 2, 417-431. MR 89k:57023

24. R. F. Williams, One-dimensional non-wandering sets, Topology 6 (1967), 473-487. MR 36 $\# 897$

Departament de Matemàtiques, Edifici Cc, Universitat Autònoma de Barcelona, 08913 Cerdanyola del Vallès, Barcelona, Spain

E-mail address: alseda@mat.uab.es

Université Lille I, Laboratoire AGat (BÂt. M2), 59655 Villeneuve D'Ascq, France (Current address: Université de Genève, Section de Mathématiques, CP 240, 2-4 Rue du Lièvre, 1211 GenÈve, Switzerland)

E-mail address: Francois.Gautero@math.unige.ch

Laboratoire de Mathématiques Emile Picard, U.M.R. CNRS 5580, Université Toulouse III, 118 route de Narbonne, 31062 Toulouse Cedex 4, France

E-mail address: guaschi@picard.ups-tlse.fr

Université Aix-Marseille I, Centre de Mathématiques et Informatique, 39 rue F. Joliot Curie, 13453 Marseille Cedex 13, France

E-mail address: los@gyptis.univ-mrs.fr

Departament de Matemàtiques, Edifici Cc, Universitat Autònoma de Barcelona, 08913 Cerdanyola del Vallès, Barcelona, Spain

E-mail address: manyosas@mat.uab.es

Departament de Matemàtica Aplicada i Anàlisi, Universitat de Barcelona, Gran Via 585, 08071-BARCELONA, SPAin

E-mail address: mumbru@mat.ub.es 